MATHEMATICS OF COMPUTATION

Volume 77, Number 264, October 2008, Pages 1941-1965

S $0025-5718(08) 02102-9$

Article electronically published on March 10, 2008

\title{
A MIXED METHOD FOR AXISYMMETRIC DIV-CURL SYSTEMS
}

\author{
DYLAN M. COPELAND, JAYADEEP GOPALAKRISHNAN, AND JOSEPH E. PASCIAK
}

\begin{abstract}
We present a mixed method for a three-dimensional axisymmetric div-curl system reduced to a two-dimensional computational domain via cylindrical coordinates. We show that when the meridian axisymmetric Maxwell problem is approximated by a mixed method using the lowest order Nédélec elements (for the vector variable) and linear elements (for the Lagrange multiplier), one obtains optimal error estimates in certain weighted Sobolev norms. The main ingredient of the analysis is a sequence of projectors in the weighted norms satisfying some commutativity properties.
\end{abstract}

\section{INTRODUCTION}

We present a mixed method for solving three-dimensional axisymmetric div-curl systems. We are interested in the case when symmetry with respect to an axis permits a reduction of the computational domain from three to two dimensions via cylindrical coordinates. The dimension reduction results in computationally efficient methods for applications in magnetostatics. However, the necessary use of cylindrical coordinates complicates the mathematical analysis of numerical methods. In particular, the coordinate transformation results in differential operators with singularities on the axis, and function spaces weighted by the radial variable are necessary. This note gives a few techniques to overcome the difficulties caused by the degenerate weights. Specifically, we construct simple interpolation operators satisfying a commuting diagram property involving a sequence of weighted spaces. This allows the application of standard techniques in the theory of mixed methods to axisymmetric div-curl systems.

An efficient method for the dimension-reduced div-curl system was recently proposed in 9], using a negative-norm least-squares technique. This method was shown to have excellent conditioning independent of jumps in the coefficient, but it allowed only for a piecewise constant coefficient. As an alternative, in this paper we present a mixed method which allows for a piecewise smooth coefficient. Another advantage is that the implementation of the mixed method involves finite element spaces more commonly used than those of the negative-norm least-squares method. The mixed method simply uses standard two-dimensional Nédélec elements [17] and

Received by the editor March 30, 2007 and, in revised form, August 29, 2007.

2000 Mathematics Subject Classification. Primary 65F10, 65N30, 78M10, 74G15, 78A30, $35 \mathrm{Q} 60$.

Key words and phrases. Mixed methods, Nedelec elements, axisymmetry, Maxwell's equations, div-curl systems, magnetostatics.

This work was supported in part by the National Science Foundation through grants DMS0713833, SCREMS-0619080, DMS-0311902, and DMS-0609544.

(C)2008 American Mathematical Society Reverts to public domain 28 years from publication 1941 
continuous piecewise linear functions. Thus the use of cylindrical coordinates does not necessitate special finite element spaces.

As a model problem, we consider Maxwell's system for magnetostatics. Let $\Omega \subset \mathbb{R}^{3}$ be a convex, simply-connected, bounded domain in $\mathbb{R}^{3}$, with a connected boundary. We assume that $\Omega$ is axisymmetric, i.e., it is invariant under rotations about the $z$-axis. Moreover, let $D$ be a polygonal domain representing the crosssection, i.e., $\Omega$ is the revolution of $D$ about the $z$-axis. We assume that $\Omega$ intersects the $z$-axis on an interval of positive length. If $\Omega$ does not intersect the $z$-axis, then the analysis of the problem is simple and standard finite element methods can be applied. The magnetostatic system is

$$
\left\{\begin{array}{lll}
\operatorname{curl} \mathbf{H} & =\mathbf{J} & \text { in } \Omega \\
\operatorname{div}(\mu \mathbf{H}) & =0 & \text { in } \Omega, \\
\mu \mathbf{H} \cdot \mathbf{n} & =0 & \text { on } \partial \Omega
\end{array}\right.
$$

where $\mathbf{H}$ is the magnetic field, $\mathbf{J}$ is the current density, $\mu$ is the magnetic permeability, and $\mathbf{n}$ is the unit outward normal on $\partial \Omega$. We assume that $\mathbf{J}$ is in $L^{2}(\Omega)^{3}$ and satisfies the compatibility condition $\operatorname{div} \mathbf{J}=0$. The coefficient $\mu$ is assumed to be piecewise smooth and to satisfy the bounds $0<\mu_{0} \leq \mu(\mathbf{x}) \leq \mu_{1}$ for all $\mathbf{x} \in \Omega$, where $\mu_{0}$ and $\mu_{1}$ are positive constants.

A standard technique to solve the magnetostatic system is via vector potentials. Define $\mathbf{B}=\mu \mathbf{H}$, the magnetic intensity, and note that $\operatorname{div} \mathbf{B}=0$. It is well known (see e.g. 11 or [10, Theorem I.3.6]) that our assumptions on $\Omega$ imply the existence of a vector potential $\mathbf{A}$ in $\mathbf{H}_{0}(\mathbf{c u r l}, \Omega)$ satisfying $\mathbf{B}=\operatorname{curl} \mathbf{A}$ and $\operatorname{div} \mathbf{A}=0$. Substituting $\mathbf{H}=\mu^{-1} \mathbf{B}$ into (1.1), we obtain the div-curl system

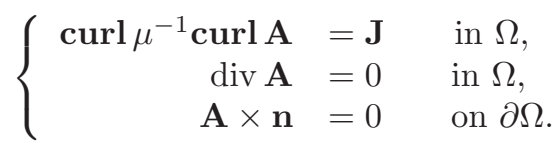

A scalar-valued function is said to be invariant under rotation if it is constant with respect to the angular variable in cylindrical coordinates, and a vector-valued function is said to be axisymmetric if its component functions in cylindrical coordinates are invariant under rotation. We assume that $\mu$ is invariant under rotation and $\mathbf{J}$ is axisymmetric. Therefore, we seek an axisymmetric solution A (cf. [5]).

It is well known that (1.2) decouples into two independent systems in the presence of axial symmetry [3. To exhibit the decoupled system, let $D$ be as before, namely $D=\{(r, z):(r, \theta, z) \in \Omega\}$, where we have used cylindrical coordinates $(r, \theta, z)$. We assume that $D$ has a polygonal boundary $\partial D$, which can be expressed as the disjoint union $\partial D=\Gamma_{0} \cup \Gamma_{1}$, where $\Gamma_{1}=\{(r, z) \in \partial D: r>0\}$. Writing the system (1.2) in cylindrical coordinates and assuming that the three components in $\mathbf{A}=A_{r} \mathbf{e}_{r}+A_{\theta} \mathbf{e}_{\theta}+A_{z} \mathbf{e}_{z}$ are $\theta$-independent, we obtain the two decoupled systems

$$
\left\{\begin{aligned}
\operatorname{curl}_{r z} \mu^{-1} \operatorname{curl}_{r z}\left(A_{r}, A_{z}\right) & =\left(J_{r}, J_{z}\right) & & \text { in } D, \\
\operatorname{div}_{r z}\left(A_{r}, A_{z}\right) \equiv \frac{1}{r} \frac{\partial}{\partial r}\left(r A_{r}\right)+\frac{\partial A_{z}}{\partial z} & =0 & & \text { in } D, \\
\left(A_{r}, A_{z}\right) \cdot\left(-n_{z}, n_{r}\right) & =0 & & \text { on } \Gamma_{1},
\end{aligned}\right.
$$

and

$$
\left\{\begin{aligned}
-\frac{\partial}{\partial r}\left(\mu^{-1} \frac{1}{r} \frac{\partial}{\partial r}\left(r A_{\theta}\right)\right)-\frac{\partial}{\partial z}\left(\mu^{-1} \frac{\partial A_{\theta}}{\partial z}\right) & =J_{\theta} & & \text { in } D, \\
A_{\theta} & =0 & & \text { on } \Gamma_{1} .
\end{aligned}\right.
$$


Here, we have used row-vector notation, $n_{r}$ and $n_{z}$ are the $r$ - and $z$-components of $\mathbf{n}$, respectively, and the two curl operators are defined by

$$
\operatorname{curl}_{r z}\left(A_{r}, A_{z}\right) \equiv \partial_{z} A_{r}-\partial_{r} A_{z} \quad \text { and } \quad \operatorname{curl}_{r z} \phi \equiv\left(-\partial_{z} \phi, \frac{1}{r} \partial_{r}(r \phi)\right),
$$

where we have abbreviated $\partial / \partial r$ to $\partial_{r}$, etc. The first system, namely (1.3), forms the so-called meridian problem, while (1.4) represents the azimuthal problem. Finite elements for such problems were considered in 8, 14, although no error analysis in the natural weighted spaces was given. The finite element suggested in [14 for the azimuthal problem was independently arrived at in [11, where a convergence analysis was also provided. Additionally, in [11, the convergence of a discretization of the azimuthal problem using a more standard element (namely, the bilinear finite element), as well as the uniform convergence of a V-cycle multigrid algorithm, was also proved. In this paper, we will analyze finite elements for the meridian problem (1.3). The main new ingredient here is an interpolation operator for a weighted $H$ (curl)-space described next.

Due to the transformation to cylindrical coordinates, the subsequent analysis of problem (1.3) involves function spaces weighted by the radial variable $r$. We denote by $L_{\alpha}^{2}(D)$ the weighted Lebesgue space of measurable functions $v$ on $D$ bounded in the norm $\|v\|_{L_{\alpha}^{2}(D)}=\left(\int_{D} r^{\alpha} v^{2} d r d z\right)^{1 / 2}$. Our notation for the natural norm on any Banach space $X$ is simply $\|\cdot\|_{X}$, but for convenience in the case of the often used space $L_{1}^{2}(D)$ (i.e., when $\alpha=1$ ), we abbreviate $\|\cdot\|_{L_{1}^{2}(D)}$ to $\|\cdot\|_{r}$. Additionally, we denote the inner product in $L_{1}^{2}(D)$ by

$$
(u, v)_{r}=\int_{D} r u v d r d z
$$

and abbreviate the norm $\|\cdot\|_{H_{1}^{1}(D)}$ to $\|\cdot\|_{1, r}$. The weighted Sobolev space $H_{\alpha}^{k}(D)$ for any $k \geq 1$ is the space of all functions in $L_{\alpha}^{2}(D)$ whose distributional derivatives of order at most $k$ are in $L_{\alpha}^{2}(D)$. We shall need the following additional weighted spaces:

$$
\begin{aligned}
\mathbf{H}_{r}(\operatorname{curl}, D) & =\left\{\mathbf{v} \in L_{1}^{2}(D)^{2}: \operatorname{curl}_{r z} \mathbf{v} \in L_{1}^{2}(D)\right\}, \\
H_{1, \diamond}^{1}(D) & =\left\{\phi \in H_{1}^{1}(D): \phi=0 \text { on } \Gamma_{1}\right\}, \\
H_{-}^{1}(D) & =\left\{\phi \in L_{-1}^{2}(D): \operatorname{grad}_{r z} \phi \in L_{1}^{2}(D)^{2}\right\} .
\end{aligned}
$$

Here $\operatorname{grad}_{r z} \phi \equiv\left(\partial_{r} \phi, \partial_{z} \phi\right)$ and the norm on $\mathbf{H}_{r}(\operatorname{curl}, D)$ is defined by

$$
\|\mathbf{v}\|_{r, \text { curl }}=\left(\|\mathbf{v}\|_{r}^{2}+\left\|\operatorname{curl}_{r z} \mathbf{v}\right\|_{r}^{2}\right)^{1 / 2} \text {. }
$$

It can be shown that the trace condition in the definition of $H_{1, \diamond}^{1}(D)$ is well defined (see 5 or Lemma A.1). These spaces have previously occurred in the literature, and we shall use their properties established in recent papers studying the axisymmetric Stokes problem [4] or the axisymmetric Maxwell system [3], as well as in earlier books [5, 13.

The remainder of the paper is outlined as follows. Section 2 is devoted to carefully defining the tangential trace operator on the weighted space $\mathbf{H}_{r}(\operatorname{curl}, D)$. In Section 3, we define and analyze a mixed formulation for (1.3) on the continuous level. The analysis proceeds by verifying the standard conditions in the theory of mixed methods [7. In Sections 4 and 5, we consider discretization and approximation based on rectangular and triangular meshes, respectively. Section 6 contains an analysis of the resulting discrete mixed formulations, simultaneously treating 
the cases of rectangular and triangular meshes. Numerical results are given in Section 7 .

\section{The tangential trace Operator}

The purpose of this section is to define the tangential trace of meridian vector fields in $\mathbf{H}_{r}$ (curl, $\left.D\right)$. This is necessary to introduce a variational formulation incorporating the boundary condition of (1.3), namely $\left(A_{r}, A_{z}\right) \cdot \mathbf{t}=0$ on $\Gamma_{1}$, where $\mathbf{t}=\left(-n_{z}, n_{r}\right)$ denotes the unit tangent vector to $\partial D$ in the $r-z$ plane, oriented counterclockwise. We will first show that smooth vector fields are dense in $\mathbf{H}_{r}(\operatorname{curl}, D)$. This will show that an integration by parts formula that holds for smooth functions also holds for $\mathbf{H}_{r}$ (curl, $D$ ) functions. The continuity of a trace operator follows.

Before proving the density result, we first set some more notation. Let $\mathcal{D}\left(\mathbb{R}^{2}\right)$ denote the space of infinitely smooth real-valued functions with compact support in $\mathbb{R}^{2}$, and set $\mathcal{D}(\bar{D})=\left\{\left.\phi\right|_{\bar{D}}: \phi \in \mathcal{D}\left(\mathbb{R}^{2}\right)\right\}$. Also, let $\mathbb{R}_{+}^{2}=\{(r, z): r>0\}$. Note that for any $w$ in $L_{1}^{2}\left(\mathbb{R}_{+}^{2}\right)$ and $\phi$ in $\mathcal{D}\left(\mathbb{R}_{+}^{2}\right)^{2}$, the action of the distribution $\operatorname{curl}_{r z} w$ on $r \phi$, denoted by $\left\langle\operatorname{curl}_{r z} w, r \phi\right\rangle$, satisfies

$$
\left\langle\operatorname{curl}_{r z} w, r \phi\right\rangle=\int_{\mathbb{R}_{+}^{2}} r w \operatorname{curl}_{r z} \phi d r d z
$$

which also equals $\left\langle r \operatorname{curl}_{r z} w, \phi\right\rangle$. Here, products with $r$ are to be considered in the sense of products of distributions with smooth functions.

Proposition 2.1. The space of smooth vector fields $\mathcal{D}(\bar{D})^{2}$ is dense in $\mathbf{H}_{r}(\mathrm{curl}, D)$.

Proof. Denoting the dual of a Hilbert space $H$ by $H^{\prime}$, we show that any functional in $\mathbf{H}_{r}$ (curl, $\left.D\right)^{\prime}$ which vanishes on $\mathcal{D}(\bar{D})^{2}$ also vanishes on $\mathbf{H}_{r}(\operatorname{curl}, D)$. The proposition then follows from Banach space theory. (This technique is also used in [10, Theorem I.2.4].) Hence, we start by considering a linear functional $l$ in $\mathbf{H}_{r}(\operatorname{curl}, D)^{\prime}$ that vanishes on $\mathcal{D}(\bar{D})^{2}$. Since $\mathbf{H}_{r}(\operatorname{curl}, D)$ is a Hilbert space, the Riesz Representation Theorem yields some $\mathbf{u}$ in $\mathbf{H}_{r}(\operatorname{curl}, D)$ such that

$$
l(\mathbf{v})=(\mathbf{u}, \mathbf{v})_{r, \text { curl }} \quad \text { for all } \mathbf{v} \text { in } \mathbf{H}_{r}(\operatorname{curl}, D),
$$

where $(\cdot, \cdot)_{r, \text { curl }}$ is the inner product in $\mathbf{H}_{r}(\operatorname{curl}, D)$ with diagonal $\|\cdot\|_{r, \text { curl }}^{2}$.

The remainder of this proof proceeds by observing the properties of the function $\mathbf{u}$ in (2.2). Let $Z: L_{1}^{2}(D) \mapsto L_{1}^{2}\left(\mathbb{R}_{+}^{2}\right)$ denote the operator that trivially extends functions by zero into $\mathbb{R}_{+}^{2}$. Also for vector functions, let $Z\left(u_{r}, u_{z}\right)=\left(Z u_{r}, Z u_{z}\right)$. Then

$$
\int_{\mathbb{R}_{+}^{2}} r\left(Z \mathbf{u} \cdot \boldsymbol{\eta}+\left(Z \operatorname{curl}_{r z} \mathbf{u}\right)\left(\operatorname{curl}_{r z} \boldsymbol{\eta}\right)\right) d r d z=l\left(\left.\boldsymbol{\eta}\right|_{D}\right)=0 \quad \text { for all } \boldsymbol{\eta} \text { in } \mathcal{D}\left(\mathbb{R}_{+}^{2}\right)^{2} .
$$

By (2.1), this implies that $r\left(Z \mathbf{u}+\operatorname{curl}_{r z} Z \operatorname{curl}_{r z} \mathbf{u}\right)=0$ in the distributional sense. But since $r Z \mathbf{u}$ is in $L_{-1}^{2}\left(\mathbb{R}_{+}^{2}\right)^{2}$, we find that the same equality must hold in $L_{-1}^{2}\left(\mathbb{R}_{+}^{2}\right)^{2}$, or equivalently,

$$
Z \mathbf{u}=-\operatorname{curl}_{r z}\left(Z \operatorname{curl}_{r z} \mathbf{u}\right) \quad \text { holds in } L_{1}^{2}\left(\mathbb{R}^{2}\right)^{2} .
$$

Next, let $\breve{\mathbf{v}}$ denote the revolution of $\mathbf{v}$, i.e., for any $\mathbf{v}$ in $L_{1}^{2}(D)$, define $\breve{\mathbf{v}}$ a.e. in $\Omega$ by $\breve{\mathbf{v}}(r, \theta, z)=v_{r}(r, z) \mathbf{e}_{r}+v_{z}(r, z) \mathbf{e}_{z}$. It is proved in 3] that for any $\mathbf{v}$ in $\mathbf{H}_{r}(\operatorname{curl}, D)$, the revolved function $\breve{\mathbf{v}}$ is in $H(\operatorname{curl}, \Omega)$. Hence for the $\mathbf{u}$ in (2.2), the 
function curl $\breve{\mathbf{u}}$ is in $L^{2}(\Omega)^{3}$. It is easy to see that if we revolve the functions on either side of (2.3) we obtain

$$
Z_{\Omega} \breve{\mathbf{u}}=-\operatorname{curl}\left(Z_{\Omega} \operatorname{curl} \breve{\mathbf{u}}\right) \quad \text { in } L^{2}\left(\mathbb{R}^{3}\right)^{3},
$$

where $Z_{\Omega}: L^{2}(\Omega)^{3} \mapsto L^{2}\left(\mathbb{R}^{3}\right)^{3}$ is the extension by zero. Thus, $Z_{\Omega}$ curl $\breve{\mathbf{u}}$ is in $\mathbf{H}\left(\operatorname{curl}, \mathbb{R}^{3}\right)$, and hence curl $\breve{\mathbf{u}}$ is in $\mathbf{H}_{0}(\operatorname{curl}, \Omega)$.

We need to show that $l(\mathbf{v})=0$ for all $\mathbf{v}$ in $\mathbf{H}_{r}(\operatorname{curl}, D)$. Since curl $\breve{\mathbf{u}}$ in $\mathbf{H}_{0}(\mathbf{c u r l}, \Omega)$ and $\breve{\mathbf{v}}$ is in $H(\mathbf{c u r l}, \Omega)$, by an integration by parts formula (see e.g. 16. Theorem 3.31]),

$$
\begin{aligned}
2 \pi l(\mathbf{v})=2 \pi(\mathbf{u}, \mathbf{v})_{r, \text { curl }} & =(\breve{\mathbf{u}}, \breve{\mathbf{v}})_{L^{2}(\Omega)^{3}}+(\operatorname{curl} \breve{\mathbf{u}}, \operatorname{curl} \breve{\mathbf{v}})_{L^{2}(\Omega)^{3}} \\
& =(\breve{\mathbf{u}}, \breve{\mathbf{v}})_{L^{2}(\Omega)^{3}}+(\operatorname{curl} \operatorname{curl} \breve{\mathbf{u}}, \breve{\mathbf{v}})_{L^{2}(\Omega)^{3}}=0 .
\end{aligned}
$$

Thus $l$ vanishes on $\mathbf{H}_{r}(\operatorname{curl}, D)$, and the proof is complete.

Next, we establish an integration by parts formula. Functions in $H_{-}^{1}(D)$ are well known to have zero trace on $\Gamma_{0}\left[3,5,11\right.$. Since $H_{-}^{1}(D)$ is a subspace of $H_{1}^{1}(D)$, and since the latter is well known to have traces in $L_{1}^{2}\left(\Gamma_{1}\right)$ (cf. [11, Proposition 2.1]), we can define the trace operator $\gamma_{t}: \mathcal{D}(\bar{D})^{2} \mapsto H_{-}^{1}(D)^{\prime}$ by

$$
\left\langle\gamma_{t}(\mathbf{v}), \phi\right\rangle=\int_{\Gamma_{1}} r \mathbf{v} \cdot \mathbf{t} \phi d s \quad \text { for all } \phi \in H_{-}^{1}(D) .
$$

Here, and elsewhere later, with slight abuse of notation we continue to use $\langle\cdot, \cdot\rangle$ for the duality pairing in different spaces. For example, in (2.5) it is the duality pairing in $H_{-}^{1}(D)$, while in (2.1) it is the duality pairing in $\mathcal{D}\left(\mathbb{R}_{+}^{2}\right)^{2}$. The space will be clear from context. The next result shows that the domain of $\gamma_{t}$ can be expanded.

Proposition 2.2. The trace operator extends to a continuous linear map from $\mathbf{H}_{r}(\operatorname{curl}, D)$ into $H_{-}^{1}(D)^{\prime}$. Moreover, the integration by parts formula

$$
\left\langle\gamma_{t}(\mathbf{v}), \phi\right\rangle=\left(\mathbf{v}, \operatorname{curl}_{r z} \phi\right)_{r}-\left(\operatorname{curl}_{r z} \mathbf{v}, \phi\right)_{r}
$$

holds for all $\mathbf{v}$ in $\mathbf{H}_{r}(\operatorname{curl}, D)$ and $\phi$ in $H_{-}^{1}(D)$.

Proof. Equation (2.6) obviously holds for all smooth functions $\mathbf{v}$ in $\mathcal{D}(\bar{D})^{2}$ and $\phi$ in $\mathcal{D}(\bar{D})$. By [11, Lemma 3.1], the set $\{\phi \in \mathcal{D}(\bar{D}): \phi$ vanishes in a neighborhood of $\left.\Gamma_{0}\right\}$ is dense in $H_{-}^{1}(D)$. Hence, for any fixed $\mathbf{v}$ in $\mathcal{D}(\bar{D})^{2}$, the identity (2.6) holds for all $\phi$ in $H_{-}^{1}(D)$. Note that here we have used the easily verifiable fact that the map $\phi \mapsto \operatorname{curl}_{r z} \phi$ is continuous from $H_{-}^{1}(D)$ to $L_{1}^{2}(D)^{2}$. Finally, by the density asserted in Proposition 2.1] the identity (2.6) holds for all $\mathbf{v}$ in $\mathbf{H}_{r}(\operatorname{curl}, D)$. The continuity of the trace operator follows by standard arguments (see e.g. the proof of [10, Theorem I.2.5]).

In the next section, we will present a mixed formulation for (1.3). The boundary condition there becomes an essential boundary condition, so we will use the subspace

$$
\mathbf{H}_{r, \diamond}(\operatorname{curl}, D)=\operatorname{ker} \gamma_{t}
$$

in our variational formulation. Clearly, by Proposition $2.2, \mathbf{H}_{r, \diamond}(\operatorname{curl}, D)$ is a closed subspace of $\mathbf{H}_{r}(\operatorname{curl}, D)$. Moreover, from (2.5) we see that smooth functions in $\operatorname{ker} \gamma_{t}$ have tangential components that vanish on $\Gamma_{1}$. 


\section{Mixed Formulation}

We can derive a mixed formulation of the meridian problem by multiplying the first two equations of (1.3) by test functions as usual and integrating by parts. The process is similar to the derivation of the standard mixed formulation [12, 16, for the three-dimensional magnetostatics equations. The appropriate integration by parts formula in weighted spaces for the curl and divergence operators are given in Proposition 2.2 and 9, Lemma 1], respectively. The resulting weak formulation seeks $\mathbf{u} \in \mathbf{H}_{r, \diamond}(\operatorname{curl}, D)$ and $p \in H_{1, \diamond}^{1}(D)$ satisfying

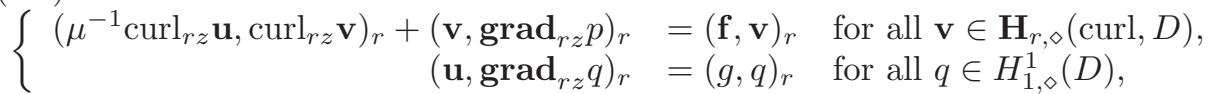

where $\mathbf{f}=\left(J_{r}, J_{z}\right)$ and $g=-\operatorname{div}_{r z}\left(A_{r}, A_{z}\right)$. Although $\left(A_{r}, A_{z}\right)$ is divergence-free in the model problem, we allow for $g$ to be any function in $L_{1}^{2}(D)$. In the remainder of this section, we quickly verify the conditions of the Babuška-Brezzi theory [7] to conclude the unique solvability of (3.1).

Defining the linear operator

$$
B: \mathbf{H}_{r, \diamond}(\operatorname{curl}, D) \rightarrow H_{1, \diamond}^{1}(D)^{\prime} \quad \text { by } \quad B \mathbf{v}(q)=\left(\mathbf{v}, \operatorname{grad}_{r z} q\right)_{r},
$$

we denote $W^{0}=\operatorname{ker} B$, i.e.

$$
W^{0}=\left\{\mathbf{v} \in \mathbf{H}_{r, \diamond}(\operatorname{curl}, D):\left(\mathbf{v}, \operatorname{grad}_{r z} q\right)_{r}=0 \text { for all } q \in H_{1, \diamond}^{1}(D)\right\} .
$$

Then we have the following theorem. In this theorem and in the remainder of the paper, the letter $C$ will denote a generic constant whose value may vary in different occurrences.

Theorem 3.1. The following holds for the axisymmetric mixed formulation (3.1):

(1) Inf-sup condition: There exists a constant $C>0$ such that

$$
C\|p\|_{1, r} \leq \sup _{\mathbf{v} \in \mathbf{H}_{r, \diamond}(\operatorname{curl}, D)} \frac{\left(\mathbf{v}, \operatorname{grad}_{r z} p\right)_{r}}{\|\mathbf{v}\|_{r, \text { curl }}}, \quad \text { for all } p \in H_{1, \diamond}^{1}(D) .
$$

(2) Coercivity on ker B: There exists a constant $C>0$ such that

$$
\|\mathbf{v}\|_{r, \text { curl }} \leq C \mu_{1}^{1 / 2}\left\|\mu^{-1 / 2} \operatorname{curl}_{r z} \mathbf{v}\right\|_{r}, \quad \text { for all } \mathbf{v} \in W^{0} .
$$

(3) Unique solvability: There is a unique $\mathbf{u}$ in $\mathbf{H}_{r, \diamond}(\operatorname{curl}, D)$ and a unique $p$ in $H_{1, \diamond}^{1}(D)$ satisfying (3.1).

Proof. (1) Denote by $\breve{H}_{0}^{1}(\Omega)$ the functions in $H_{0}^{1}(\Omega)$ invariant under rotation. The mapping $\breve{H}_{0}^{1}(\Omega) \rightarrow H_{1, \diamond}^{1}(D)$ defined by $v(r, z)=\breve{v}(r, \theta, z)$ is an isomorphism (see $\S I I .4$ of [5]). Applying this isomorphism and the Poincaré inequality on $H_{0}^{1}(\Omega)$ we get

$$
2 \pi\|p\|_{1, r}^{2} \leq\|\breve{p}\|_{H^{1}(\Omega)}^{2} \leq C\|\operatorname{grad} \breve{p}\|_{L^{2}(\Omega)}^{2}=2 \pi C\left\|\operatorname{grad}_{r z} p\right\|_{r}^{2},
$$

where $\operatorname{grad}(\cdot)$ denotes the three-dimensional gradient operator. Note that for any $p$ in $H_{1, \diamond}^{1}(D)$, the gradient $\operatorname{grad} \breve{p}$ is in $\mathbf{H}_{0}(\operatorname{curl}, \Omega)$ and hence $\operatorname{grad}_{r z} p$ is in $\mathbf{H}_{r, \diamond}(\operatorname{curl}, D)$ (cf. [3, Proposition 3.19]). Moreover, $\operatorname{curl}_{r z}\left(\operatorname{grad}_{r z} p\right)=0$ implies

$$
C\|p\|_{1, r} \leq \frac{\left(\operatorname{grad}_{r z} p, \operatorname{grad}_{r z} p\right)_{r}}{\left\|\operatorname{grad}_{r z} p\right\|_{r, \operatorname{curl}}} \leq \sup _{\mathbf{v} \in \mathbf{H}_{r, \diamond}(\operatorname{curl}, D)} \frac{\left(\mathbf{v}, \operatorname{grad}_{r z} p\right)_{r}}{\|\mathbf{v}\|_{r, \text { curl }}} .
$$


(2) Let $\mathbf{v} \in W^{0}$ and consider the revolved vector field $\breve{\mathbf{v}}=v_{r} \mathbf{e}_{r}+v_{z} \mathbf{e}_{z}$ defined in $\Omega$. Clearly $\breve{\mathbf{v}} \times \mathbf{n}=0$ on $\partial \Omega$. By [3, Proposition 3.19], $\breve{\mathbf{v}}$ is in $H(\operatorname{curl}, \Omega)$. Let us now also show that

$$
(\breve{\mathbf{v}}, \operatorname{grad} q)_{L^{2}(\Omega)}=0, \quad \text { for all } q \in H_{0}^{1}(\Omega) .
$$

The left hand side is

$$
\begin{aligned}
(\breve{\mathbf{v}}, \operatorname{grad} q)_{L^{2}(\Omega)} & =\iint_{D} \int_{0}^{2 \pi} r \breve{\mathbf{v}} \cdot \operatorname{grad} q d \theta d r d z \\
& =\iint_{D} \int_{0}^{2 \pi} r\left(v_{r} \partial_{r} q+v_{z} \partial_{z} q\right) d \theta d r d z \\
& =2 \pi \iint_{D} r\left(v_{r} \partial_{r} q+v_{z} \partial_{z} q\right) d r d z
\end{aligned}
$$

where

$$
q(r, z)=\frac{1}{2 \pi} \int_{0}^{2 \pi} q(r, \theta, z) d \theta
$$

and the interchanges in the order of integration and differentiations are justified as usual. Since $q$ is in $H_{0}^{1}(\Omega)$, by [5, Theorem II.3.1], the averaged function $q$ is in $H_{1, \diamond}^{1}(D)$. Consequently, the above calculation implies that

$$
(\breve{\mathbf{v}}, \operatorname{grad} q)_{L^{2}(\Omega)}=2 \pi\left(\mathbf{v}, \operatorname{grad}_{r z} q\right)_{r}=0,
$$

as $\mathbf{v}$ is given to be in $W^{0}$. By a standard Poincaré-Friedrichs estimate [1, 10, (sometimes also called Weber inequality [3, 18]) for functions satisfying (3.3), we have

$$
\|\breve{\mathbf{v}}\|_{L^{2}(\Omega)} \leq C\|\operatorname{curl} \breve{\mathbf{v}}\|_{L^{2}(\Omega)} .
$$

Since $\breve{\mathbf{v}}$ is axisymmetric, $\operatorname{curl} \breve{\mathbf{v}}=\left(\operatorname{curl}_{r z} \mathbf{v}\right) \mathbf{e}_{\theta}$, so the above inequality implies a corresponding estimate on $D$, namely

$$
\|\mathbf{v}\|_{r} \leq C\left\|\operatorname{curl}_{r z} \mathbf{v}\right\|_{r} \leq C \mu_{1}^{1 / 2}\left\|\mu^{-1 / 2} \operatorname{curl}_{r z} \mathbf{v}\right\|_{r} .
$$

This is equivalent to the desired inequality.

(3) The final statement follows by applying the Babuška-Brezzi theory for mixed systems, as the previous two statements of the theorem verified the assumptions required to apply the theory.

\section{Approximation on rectangular meshes}

In this section, we introduce finite element spaces based on rectangular meshes, which approximate the spaces $\mathbf{H}_{r}(\operatorname{curl}, D)$ and $H_{1}^{1}(D)$. Triangular finite elements are given in the next section. Although triangular elements cover more general domains, we include the rectangular case, because its analysis readily reveals the main ideas, and they are simple to implement when applicable.

The rectangular finite element functions are simple bilinear functions for $H_{1}^{1}(D)$ and the two-dimensional edge-based Nédélec functions for $\mathbf{H}_{r}(\operatorname{curl}, D)$. To establish notation for our finite element spaces, first define the polynomial spaces

$$
\begin{aligned}
Q_{1,1} & =\left\{p(r, z)=c_{0}+c_{1} r+c_{2} z+c_{3} r z: c_{i} \in \mathbb{R} \text { for } 0 \leq i \leq 3\right\}, \\
\mathcal{N D}_{1,1} & =\{(a+b z, c+d r): a, b, c, d \in \mathbb{R}\} .
\end{aligned}
$$


Given a rectangular mesh $\mathcal{T}_{h}$ aligned with the coordinate axes, define the finite element spaces

$$
\begin{aligned}
V_{h} & =\left\{u \in H_{1}^{1}(D):\left.u\right|_{K} \in Q_{1,1} \text { for all } K \in \mathcal{T}_{h}\right\}, \\
W_{h} & =\left\{\mathbf{v} \in \mathbf{H}_{r}(\operatorname{curl}, D):\left.\mathbf{v}\right|_{K} \in \mathcal{N D}_{1,1} \text { for all } K \in \mathcal{T}_{h}\right\}, \\
S_{h} & =\left\{u \in L_{1}^{2}(D):\left.u\right|_{K} \text { is constant, for all } K \in \mathcal{T}_{h}\right\} .
\end{aligned}
$$

Additionally, let

$$
\begin{aligned}
V_{h, \diamond} & =\left\{v \in V_{h}: v=0 \text { on } \Gamma_{1}\right\}, \\
W_{h, \diamond} & =\left\{\mathbf{v} \in W_{h}: \mathbf{v} \cdot \mathbf{t}=0 \text { on } \Gamma_{1}\right\} .
\end{aligned}
$$

Our aim in this section is to construct projectors $\Pi_{h}^{V}, \Pi_{h}^{W}$ and $\Pi_{h}^{S}$ onto the above finite element spaces such that the following diagram commutes:

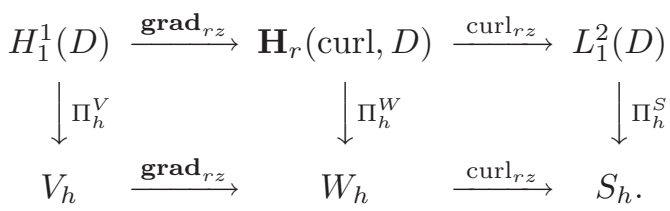

The above is somewhat of an abuse of notation, as the first two projectors are defined on sufficiently smooth functions in $H_{1}^{1}(D)$ and $\mathbf{H}_{r}(\operatorname{curl}, D)$, respectively. The importance of such a property in the analysis of mixed methods with the usual (unweighted) Sobolev spaces is well known. As in the case of the standard unweighted Sobolev spaces, it is quite technical to construct projectors whose domain is the entire corresponding Sobolev space appearing in (4.2). Hence, the projectors we construct will require further regularity.

We first define the interpolation operator $\Pi_{h}^{V}$ by its action element by element. On every element $K$ whose boundary does not intersect $\Gamma_{0},\left.\left(\Pi_{h}^{V} v\right)\right|_{K}$ is the unique bilinear function whose value at each of the four vertices of $K$ coincides with that of $v$. The remaining elements have vertices of the form $\left(0, z_{0}\right),\left(h_{r}, z_{0}\right),\left(h_{r}, z_{1}\right)$, and $\left(0, z_{1}\right)$, with $z_{1}=z_{0}+h_{z}$. On these elements, we define new degrees of freedom by

$$
\begin{array}{ll}
m_{1}(v)=v\left(h_{r}, z_{0}\right), \quad m_{2}(v)=\int_{0}^{h_{r}} r \partial_{r} v\left(r, z_{0}\right) d r, \\
m_{3}(v)=v\left(h_{r}, z_{1}\right), \quad m_{4}(v)=\int_{0}^{h_{r}} r \partial_{r} v\left(r, z_{1}\right) d r .
\end{array}
$$

The interpolant $\Pi_{h}^{V} v$ on such an element is the unique function in $Q_{1,1}$ satisfying $m_{i}\left(\Pi_{h}^{V} v\right)=m_{i}(v)$ for $i=1, \ldots, 4$.

Remark 4.1. The definition and analysis of the above interpolant are very similar to those of an interpolation operator defined in [11, where the weight $r^{1 / 2}$ was used in place of $r$ in defining $m_{2}$ and $m_{4}$. We chose the $r$ weight for convenience. A similar analysis could be provided for the $r^{1 / 2}$ weight. In [11, the reason for the introduction of integral degrees of freedom was (incorrectly) given to be the lack of continuity of the functional $v \mapsto v(0, z)$ in $H_{1}^{2}(D)$. However, this functional is indeed continuous in $H_{1}^{2}(D)$, as shown in [15, Theorem 4.7]. Relying on this continuity, an alternate approach to approximation theory in $H_{1}^{2}(D)$ is pursued in [15]. However, [15] does not give the other projectors and the commutativity properties in (4.2) needed for the analysis of mixed methods. 
Next, we define the projection $\Pi_{h}^{W}$ onto $W_{h}$. On rectangles $K$ not intersecting $\Gamma_{0}$, $\Pi_{h}^{W}$ is the standard Nédélec interpolant defined by the unweighted integrals of the tangential components along edges, i.e., the degrees of freedom are $\int_{e}\left(\Pi_{h}^{W} \mathbf{v}\right) \cdot \mathbf{t} d s$ for all edges $e$ of $K$. On the remaining rectangles, the degrees of freedom are

$$
\begin{array}{ll}
M_{1}(\mathbf{v})=\int_{\left(0, z_{0}\right)}^{\left(h_{r}, z_{0}\right)} r \mathbf{v} \cdot \mathbf{t} d s, & M_{2}(\mathbf{v})=\int_{\left(h_{r}, z_{0}\right)}^{\left(h_{r}, z_{0}+h_{z}\right)} \mathbf{v} \cdot \mathbf{t} d s, \\
M_{3}(\mathbf{v})=\int_{\left(h_{r}, z_{0}+h_{z}\right)}^{\left(0, z_{0}+h_{z}\right)} r \mathbf{v} \cdot \mathbf{t} d s, & M_{4}(\mathbf{v})=\int_{K} r \operatorname{curl}_{r z} \mathbf{v} d r d z .
\end{array}
$$

Here $\int_{\mathbf{a}_{1}}^{\mathbf{a}_{2}}$ denotes the straight line integral from point $\mathbf{a}_{1}$ to point $\mathbf{a}_{2}$ in the $r-z$ plane. For any function $\mathbf{v}$ for which the moments $M_{i}$ exist, we define $\left.\Pi_{h}^{W} \mathbf{v}\right|_{K}$ as a function in $\mathcal{N D}_{1,1}$ satisfying $M_{i}\left(\Pi_{h}^{W} \mathbf{v}\right)=M_{i}(\mathbf{v})$ for $i=1, \ldots, 4$. That $\Pi_{h}^{W} \mathbf{v}$ is uniquely defined this way follows from the next lemma.

Finally, for the space $S_{h}$ at the end of (4.2), we define $\Pi_{h}^{S} w$ for any $w$ in $L_{1}^{2}(D)$ as the unique function in $S_{h}$ such that on a mesh element $K$, its restriction $\left.\left(\Pi_{h}^{S} w\right)\right|_{K}$ satisfies

$$
\begin{aligned}
\int_{K} r\left(\Pi_{h}^{S} w\right) v d r d z & =\int_{K} r w v d r d z & \text { if } \bar{K} \text { intersects } \Gamma_{0}, \text { and } \\
\int_{K}\left(\Pi_{h}^{S} w\right) v d r d z=\int_{K} w v d r d z & & \text { otherwise, }
\end{aligned}
$$

for all $v$ in $S_{h}$. With these definitions, we have the following lemma verifying the commutative properties in (4.2).

Lemma 4.1. The interpolation operators defined above have the following properties:

(1) The degrees of freedom in (4.3) are continuous linear functionals on $H_{1}^{2}(D)$ and are unisolvent. Hence $\Pi_{h}^{V}$ is well defined and continuous on $H_{1}^{2}(D)$.

(2) The degrees of freedom in (4.4) are continuous linear functionals on $H_{1}^{1}(D)^{2}$ and are unisolvent. Hence $\Pi_{h}^{W}$ is well defined and continuous on $H_{1}^{1}(D)^{2}$.

(3) The commutative properties

$$
\begin{aligned}
\operatorname{curl}_{r z} \Pi_{h}^{W} \mathbf{v} & =\Pi_{h}^{S} \operatorname{curl}_{r z} \mathbf{v}, \\
\Pi_{h}^{W} \operatorname{grad}_{r z} u & =\operatorname{grad}_{r z} \Pi_{h}^{V} u,
\end{aligned}
$$

hold for any $u$ and $\mathbf{v}$ for which all of the above quantities are bounded.

(4) $\Pi_{h}^{V}$ and $\Pi_{h}^{W}$ preserve zero boundary conditions, i.e., if $u$ is in $H_{1, \diamond}^{1}(D) \cap$ $H_{1}^{2}(D)$, then $\Pi_{h}^{V}$ is in $V_{h, \diamond}$, and if $\mathbf{v}$ is in $\mathbf{H}_{r, \diamond}(\operatorname{curl}, D) \cap H_{1}^{1}(D)^{2}$, then $\Pi_{h}^{W} \mathbf{v}$ is in $W_{h, \diamond}$.

Proof. The continuity of $m_{1}$ and $m_{3}$ on $H_{1}^{2}(D)$ follows from the standard Sobolev inequality (because the points involved are away from the $z$-axis). For $m_{2}$ and $m_{4}$, the same continuity follows from [11, Proposition 2.1] or Lemma A.1 Similarly, $M_{i}$ is continuous on $H_{1}^{1}(D)^{2}$ for $1 \leq i \leq 4$.

Next, let us prove the unisolvence of $\left\{M_{i}\right\}$. (We omit the proof of unisolvence of $\left\{m_{i}\right\}$, as it is simpler.) Suppose $\mathbf{v}_{h}=(a+b z, c+d r)$ on a rectangle $K$ intersecting $\Gamma_{0}$, with $M_{i}\left(\mathbf{v}_{h}\right)=0$ for $1 \leq i \leq 4$. We need to show that $\mathbf{v}_{h}$ vanishes. Since

$$
\operatorname{curl}_{r z} \mathbf{v}_{h}=b-d,
$$


$M_{4}\left(\mathbf{v}_{h}\right)=0$ implies $b=d$. Consequently, $\mathbf{v}_{h}=\operatorname{grad}_{r z} \psi_{h}$ for some bilinear function $\psi_{h}$ in $Q_{1,1}$. It follows from $M_{1}\left(\mathbf{v}_{h}\right)=0$ and $M_{3}\left(\mathbf{v}_{h}\right)=0$ that $\partial \psi_{h} / \partial r$ vanishes on the horizontal edges of $K$. Thus, $\psi_{h}(r, z)=c_{0}+c_{2} z$. Now

$$
\int_{\left(h_{r}, z_{0}\right)}^{\left(h_{r}, z_{0}+h_{z}\right)} \operatorname{grad}_{r z} \psi_{h} \cdot \mathbf{t} d s=M_{2}\left(\mathbf{v}_{h}\right)=0
$$

implies $\psi_{h}(r, z)=c_{0}$ is constant, i.e., $\mathbf{v}_{h}=\operatorname{grad}_{r z} \psi_{h}=0$.

The commutative properties follow, in a standard way, elements away from $\Gamma_{0}$. For the remaining elements, the identity $\operatorname{curl}_{r z} \Pi_{h}^{W} \mathbf{v}=\Pi_{h}^{S} \operatorname{curl}_{r z} \mathbf{v}$ follows immediately from the definition of $M_{4}$, since $\operatorname{curl}_{r z} \mathbf{w}$ is a scalar constant for any $\mathbf{w}$ in $\mathcal{N} \mathcal{D}_{1,1}$. To verify the second identity $\Pi_{h}^{W} \operatorname{grad}_{r z} u=\operatorname{grad}_{r z} \Pi_{h}^{V} u$, it suffices to show that the degrees of freedom $M_{i}$ agree for $\operatorname{grad}_{r z} u$ and $\operatorname{grad}_{r z} \Pi_{h}^{V} u$, because the gradient of any function in $V_{h}$ is in $W_{h}$. By the definition (4.3) of $\Pi_{h}^{V}$,

$$
\begin{aligned}
M_{1}\left(\operatorname{grad}_{r z}\left(u-\Pi_{h}^{V} u\right)\right) & =\int_{0}^{h_{r}} r\left(\partial_{r} u\left(r, z_{0}\right)-\partial_{r} \Pi_{h}^{V} u\left(r, z_{0}\right)\right) d r=0, \\
M_{2}\left(\operatorname{grad}_{r z}\left(u-\Pi_{h}^{V} u\right)\right) & =\int_{z_{0}}^{z_{0}+h_{z}}\left(\partial_{z} u\left(h_{r}, z\right)-\partial_{z} \Pi_{h}^{V} u\left(h_{r}, z\right)\right) d z \\
& =u\left(h_{r}, z_{0}+h_{z}\right)-\Pi_{h}^{V} u\left(h_{r}, z_{0}+h_{z}\right)-u\left(h_{r}, z_{0}\right)+\Pi_{h}^{V} u\left(h_{r}, z_{0}\right) \\
& =0, \\
M_{3}\left(\operatorname{grad}_{r z}\left(u-\Pi_{h}^{V} u\right)\right) & =\int_{h_{r}}^{0}-r\left(\partial_{r} u\left(r, z_{0}+h_{z}\right)-\partial_{r} \Pi_{h}^{V} u\left(r, z_{0}+h_{z}\right)\right) d r=0, \\
M_{4}\left(\operatorname{grad}_{r z}\left(u-\Pi_{h}^{V} u\right)\right) & =\int_{K} r \operatorname{curl}_{r z} \operatorname{grad}_{r z}\left(u-\Pi_{h}^{V} u\right) d r d z=0 .
\end{aligned}
$$

This proves the second commutative identity.

Finally, the last assertion on the boundary preservation properties is obvious by construction.

We conclude this section by proving approximation properties for the interpolation operators. From now on, whenever we use the generic constant $C$, its value will always be independent of the mesh size $h=\max _{K \in \mathcal{T}_{h}} \operatorname{diam}(K)$.

Lemma 4.2 (Approximation estimates). There exists a constant $C$ such that

$$
\begin{aligned}
\left\|\operatorname{grad}_{r z}\left(u-\Pi_{h}^{V} u\right)\right\|_{L_{1}^{2}(D)} & \leq C h|u|_{H_{1}^{2}(D)}, \\
\left\|\mathbf{v}-\Pi_{h}^{W} \mathbf{v}\right\|_{L_{1}^{2}(D)^{2}} & \leq C h|\mathbf{v}|_{H_{1}^{1}(D)^{2}}, \\
\left\|\operatorname{curl}_{r z}\left(\mathbf{v}-\Pi_{h}^{W} \mathbf{v}\right)\right\|_{L_{1}^{2}(D)} & \leq C h\left|\operatorname{curl}_{r z} \mathbf{v}\right|_{H_{1}^{1}(D)}, \\
\left\|w-\Pi_{h}^{S} w\right\|_{L_{1}^{2}(D)} & \leq C h|w|_{H_{1}^{1}(D)},
\end{aligned}
$$

for all $u \in H_{1}^{2}(D), w \in H_{1}^{1}(D)$, and $\mathbf{v} \in H_{1}^{1}(D)^{2}$. For (4.8), we additionally require $\operatorname{curl}_{r z} \mathbf{v} \in H_{1}^{1}(D)$.

Proof. These estimates are established by proving analogous local estimates on each element, squaring them, and summing. For elements away from the $z$-axis, standard arguments prove the estimates. For the remaining elements $K$ intersecting $\Gamma_{0}$, we will now show that all of the estimates follow from the fact that whenever $\phi$ in 
$H_{1}^{1}(K)$ satisfies any one of the conditions

$$
\begin{aligned}
& \int_{e} r \phi(r, z) d r=0, \quad \text { for an edge } e \text { of } K \text { having a vertex in } \Gamma_{0}, \text { or } \\
& \int_{e} \phi(r, z) d r=0, \quad \text { for the vertical edge } e \text { of } K \text { not intersecting } \Gamma_{0}, \text { or }
\end{aligned}
$$

$$
\int_{K} r \phi(r, z) d r d z=0
$$

then

$$
\|\phi\|_{L_{1}^{2}(K)} \leq C h|\phi|_{H_{1}^{1}(K)} .
$$

The fact that (4.13) holds under conditions (4.10) and (4.11) is contained in 11, Lemma 2.1]. Its proof under condition (4.12) is very similar, so we omit it.

Consider the last operator $\Pi_{h}^{S}$ first. Since $\phi=\left.\left(w-\Pi_{h}^{S} w\right)\right|_{K}$ satisfies (4.12), the estimate $\|\phi\|_{L_{1}^{2}(K)} \leq C h|w|_{H_{1}^{1}(K)}$ follows from (4.13) and $\left|\Pi_{h}^{S} w\right|_{H_{1}^{1}(K)}=0$. This proves (4.9). To prove estimate (4.7) for $\Pi_{h}^{W}$, let $\left(\phi_{r}, \phi_{z}\right)=\mathbf{v}-\Pi_{h}^{W} \mathbf{v}$ and observe that by construction, $\phi_{r}$ satisfies (4.10) and $\phi_{z}$ satisfies (4.11). Hence

$$
\left\|\phi_{r}\right\|_{L_{1}^{2}(K)} \leq C h\left|\phi_{r}\right|_{H_{1}^{1}(K)}, \quad\left\|\phi_{z}\right\|_{L_{1}^{2}(K)} \leq C h\left|\phi_{z}\right|_{H_{1}^{1}(K)} .
$$

It is easy to verify by direct calculation that $\left|\Pi_{h}^{W} \mathbf{v}\right|_{H_{1}^{1}(K)^{2}} \leq C|\mathbf{v}|_{H_{1}^{1}(K)^{2}}$. Using this to bound the right hand sides above, we get (4.7). Estimate (4.8) follows from Lemma 4.1 because

$$
\begin{aligned}
\left\|\operatorname{curl}_{r z}\left(\mathbf{v}-\Pi_{h}^{W} \mathbf{v}\right)\right\|_{L_{1}^{2}(D)} & =\left\|\operatorname{curl}_{r z} \mathbf{v}-\Pi_{h}^{S} \operatorname{curl}_{r z} \mathbf{v}\right\|_{L_{1}^{2}(D)} & & \text { by Lemma 4.1(3), } \\
& \leq C h\left|\operatorname{curl}_{r z} \mathbf{v}\right|_{H_{1}^{1}(D)} & & \text { by (4.9). }
\end{aligned}
$$

Proof of the final estimate proceeds similarly using Lemma 4.1

$$
\begin{aligned}
\left\|\operatorname{grad}_{r z}\left(u-\Pi_{h}^{V} u\right)\right\|_{L_{1}^{2}(D)} & =\left\|\operatorname{grad}_{r z} u-\Pi_{h}^{W} \operatorname{grad}_{r z} u\right\|_{L_{1}^{2}(D)} & & \text { by Lemma 4.1](3), } \\
& \leq C h\left|\operatorname{grad}_{r z} u\right|_{H_{1}^{1}(D)} & & \text { by (4.7), }
\end{aligned}
$$

from which (4.6) follows.

\section{Approximation on triangular meshes}

We now consider triangular meshes, proving results analogous to those given in the previous section for rectangular meshes. In particular, we want to construct projectors onto triangular finite element spaces satisfying the commuting diagram property (4.2) and possessing optimal approximation properties.

With triangular elements, we are now able to mesh more general polygonal domains $D$. Let $\mathcal{T}_{h}$ be a mesh of $D$ consisting of triangles, satisfying the usual finite element assumptions. For simplicity, we assume that $\mathcal{T}_{h}$ is quasiuniform. Define the polynomial spaces

$$
\begin{aligned}
P_{1} & =\left\{p(r, z)=c_{0}+c_{1} r+c_{2} z: c_{i} \in \mathbb{R} \text { for } 0 \leq i \leq 2\right\}, \\
\mathcal{N D}_{1} & =\{(a-b z, c+b r): a, b, c \in \mathbb{R}\} .
\end{aligned}
$$


Then for each $\mathbf{v}$ in $\mathcal{N} \mathcal{D}_{1}(\tau), \mathbf{v} \cdot \mathbf{t}$ is constant along each edge of $\tau$. The global finite element spaces are

$$
\begin{aligned}
V_{h} & =\left\{u \in H_{1}^{1}(D):\left.u\right|_{\tau} \in P_{1} \text { for all } \tau \in \mathcal{T}_{h}\right\}, \\
W_{h} & =\left\{\mathbf{v} \in \mathbf{H}_{r}(\operatorname{curl}, D):\left.\mathbf{v}\right|_{\tau} \in \mathcal{N} \mathcal{D}_{1} \text { for all } \tau \in \mathcal{T}_{h}\right\}, \\
S_{h} & =\left\{u \in L_{1}^{2}(D):\left.u\right|_{\tau} \in \mathbb{R} \text { is constant, for all } \tau \in \mathcal{T}_{h}\right\} .
\end{aligned}
$$

One obvious set of global degrees of freedom for $V_{h}$ consists of the function values at all mesh vertices. However, we cannot consider the nodal interpolant corresponding to these degrees of freedom, as the values on the $z$-axis are undefined for functions in our weighted spaces. As in the rectangular case, we therefore define new degrees of freedom through weighted edge moments. In the triangular case, there is an additional complication due to multiple interior edges meeting at a vertex on the $z$-axis. Hence, we associate with each vertex $\mathbf{a}_{i}$ in $\Gamma_{0}$ one edge $e\left(\mathbf{a}_{i}\right)$ such that $\mathbf{a}_{i}$ is an endpoint of $e\left(\mathbf{a}_{i}\right)$ and $e\left(\mathbf{a}_{i}\right) \nsubseteq \Gamma_{0}$. If $\mathbf{a}_{i}$ is an endpoint of $\Gamma_{0}$, then we choose $e\left(\mathbf{a}_{i}\right)$ to be the mesh edge connected to $\mathbf{a}_{i}$ lying on $\Gamma_{1}$. Then, we set one global degree of freedom per mesh vertex by

$$
\begin{cases}\sigma_{i}^{V}(u)=u\left(\mathbf{a}_{i}\right), & \text { if } \mathbf{a}_{i} \notin \Gamma_{0}, \\ \sigma_{i}^{V}(u)=\int_{e\left(\mathbf{a}_{i}\right)} r \mathbf{t} \cdot \operatorname{grad}_{r z} u d s, & \text { if } \mathbf{a}_{i} \in \Gamma_{0} .\end{cases}
$$

Proposition 5.1. The linear functionals in (5.1) are continuous on $H_{1}^{2}(D)$ and form unisolvent degrees of freedom for $V_{h}$.

Proof. For $\mathbf{a}_{i} \notin \Gamma_{0}$, by a standard Sobolev inequality,

$$
\left|\sigma_{i}^{V}(u)\right|=\left|u\left(\mathbf{a}_{i}\right)\right| \leq c_{i}\|u\|_{H_{1}^{2}(D)},
$$

while for $\mathbf{a}_{i} \in \Gamma_{0}$, by Lemma A.1.

$$
\left|\sigma_{i}^{V}(u)\right|=\left|\int_{e\left(\mathbf{a}_{i}\right)} r \mathbf{t} \cdot \operatorname{grad}_{r z} u d s\right| \leq c_{i}\|u\|_{H_{1}^{2}(D)},
$$

for some constants $c_{i}$ independent of $u$. Hence, the degrees of freedom are continuous linear functionals on $H_{1}^{2}(D)$. It only remains to show that these degrees of freedom are unisolvent. If $v_{h} \in V_{h}$ has $\sigma_{i}^{V}\left(v_{h}\right)=0$ for all $i$, then obviously $v_{h}$ vanishes at all vertices $\mathbf{a}_{i} \notin \Gamma_{0}$. It also vanishes on the remaining vertices $\mathbf{a}_{i} \in \Gamma_{0}$ because the zero integral degrees of freedom imply that $v_{h}\left(\mathbf{a}_{i}\right)$ must equal the value of $v_{h}$ at the other endpoint of $e\left(\mathbf{a}_{i}\right)$. Hence $v_{h} \equiv 0$.

Next, we define the set of global degrees of freedom for the Nédélec space $W_{h}$ as

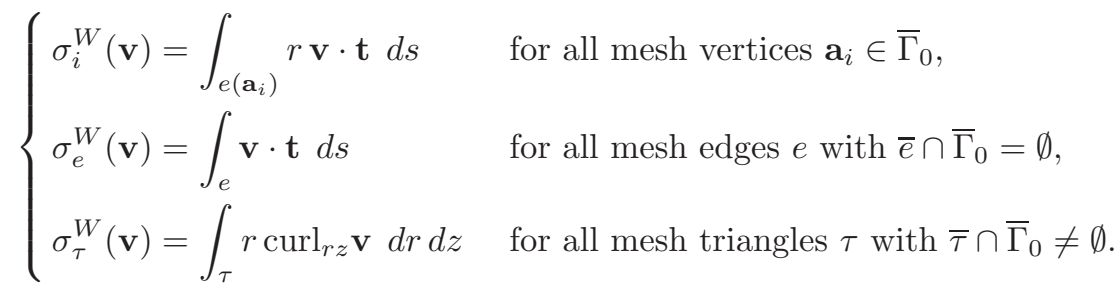

Note that these differ from the standard Nédélec degrees of freedom [17] in three respects: (i) We use weighted edge moments near $\Gamma_{0}$. (ii) Not all interior edges are used in defining the degrees of freedom. (iii) Elements near $\Gamma_{0}$ have an interior 
degree of freedom even though we are only considering the lowest order case. Due to these differences, we must now verify that the new degrees of freedom control $W_{h}$.

Proposition 5.2. The linear functionals in (5.2) are continuous on $H_{1}^{1}(D)^{2}$ and form unisolvent degrees of freedom for $W_{h}$.

Proof. To prove unisolvence, suppose that all the degrees of freedom in (5.2) vanish for a function $\mathbf{v}_{h}$ in $W_{h}$. Then, clearly $\mathbf{v}_{h}$ vanishes on elements $\tau$ not intersecting $\Gamma_{0}$. It remains to show that $\mathbf{v}_{h}$ vanishes on the remaining elements, which we collect into a set $S$. The elements in $S$ can be classified in two types: for $k=1$ or 2 , we say that a triangle $\tau$ in $S$ is of type $k$ if $\tau$ has exactly $k$ vertices on $\Gamma_{0}$ (see Figure 1). For any $\tau \in S$, writing $\left.\mathbf{v}_{h}\right|_{\tau}=(a-b z, c+b r)$ and using the fact that the interior degree of freedom is zero, we have

$$
-2 b \int_{\tau} r d r d z=\int_{\tau} r \operatorname{curl}_{r z}(a-b z, c+b r) d r d z=\int_{\tau} r \operatorname{curl}_{r z} \mathbf{v}_{h} d r d z=0 .
$$

Hence $b=0$ and $\mathbf{v}_{h}$ is constant on $\tau$. Therefore, to prove that $\mathbf{v}_{h}$ vanishes on any $\tau$ in $S$, it suffices to show that the tangential component of $\mathbf{v}_{h}$ is zero on any two edges of $\tau$.

Note that around any interior vertex of $\Gamma_{0}$, there are two triangles of type two separated by a number (possibly none) of type one triangles. The case of an end point is similar but only involves one triangle of type two.

Now let us prove that $\mathbf{v}_{h}$ vanishes on all type one triangles in $S$. Let $\tau$ be a type one triangle and let $\boldsymbol{a}$ be its vertex on $\bar{\Gamma}_{0}$ (including the endpoints of $\Gamma_{0}$ ). Then, $e(\boldsymbol{a})$ cannot be an edge shared by type two triangles only, i.e., there is at least one type one triangle, say $\tau^{\prime}$, having $e(\boldsymbol{a})$ as an edge (see Figure 1). This triangle $\tau^{\prime}$ need not coincide with $\tau$. It has an edge not intersecting $\Gamma_{0}$ where the tangential component of $\mathbf{v}_{h}$ is given to be zero. Therefore, $\mathbf{v}_{h} \cdot \mathbf{t}$ is zero on two edges of $\tau^{\prime}$, hence $\mathbf{v}_{h}$ vanishes on $\tau^{\prime}$. We can repeat this argument on any type one triangle adjacent to $\tau^{\prime}$ (since $\mathbf{v}_{h} \cdot \mathbf{t}=0$ on all edges of $\tau^{\prime}$ ). Therefore $\mathbf{v}_{h}$ vanishes on all type one triangles connected to $\boldsymbol{a}$, including $\tau$.

Next, consider a type two triangle $\tau$. It has two edges not on $\Gamma_{0}$. We claim that $\mathbf{v}_{h} \cdot \mathbf{t}$ vanishes on these two edges. Indeed, if $e$ is such an edge, and $e$ is shared by a type one triangle, then by the conclusion of the previous paragraph, $\mathbf{v}_{h} \cdot \mathbf{t}=0$ on $e$. Also, if $e$ is on $\Gamma_{1}$, then obviously $\mathbf{v}_{h} \cdot \mathbf{t}=0$ on $e$. The only other possibility is that $e$ is shared by another type two triangle $\tau^{\prime}$. But in this case, $e$ coincides with $e(\boldsymbol{a})$ where $\boldsymbol{a}$ is the common vertex of $\tau$ and $\tau^{\prime}$ on $\Gamma_{0}$. Obviously, $\mathbf{v}_{h} \cdot \mathbf{t}=0$ on $e(\boldsymbol{a})$ since all degrees of freedom are given to be zero. Thus, $\mathbf{v}_{h} \cdot \mathbf{t}$ vanishes on two edges of each type two triangle, so $\mathbf{v}_{h}$ vanishes on type two triangles as well.

The continuity of the linear functionals on $H_{1}^{1}(D)^{2}$ follows easily from Lemma A.1 and standard results.

Define the projector $\Pi_{h}^{V}$ onto $V_{h}$ by

$$
\sigma_{i}^{V}\left(\Pi_{h}^{V} v\right)=\sigma_{i}^{V}(v)
$$

for all the degrees of freedom $\sigma_{i}^{V}$ in (5.1). Similarly define the projector $\Pi_{h}^{W}$ onto $W_{h}$ using all the degrees of freedom in (5.2). By Propositions 5.1 and 5.2, these projectors are well defined. In particular, $\Pi_{h}^{W} \mathbf{u}$ for any $\mathbf{u}$ in $H_{1}^{1}(D)^{2}$ automatically satisfies the tangential continuity constraints of $W_{h}$ along all mesh edges even 


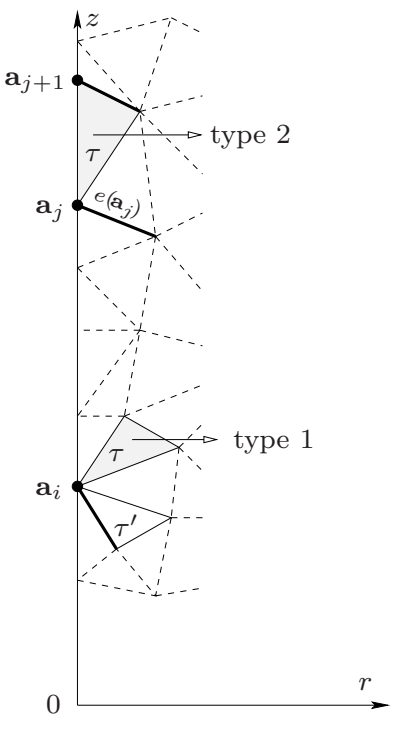

$(a)$
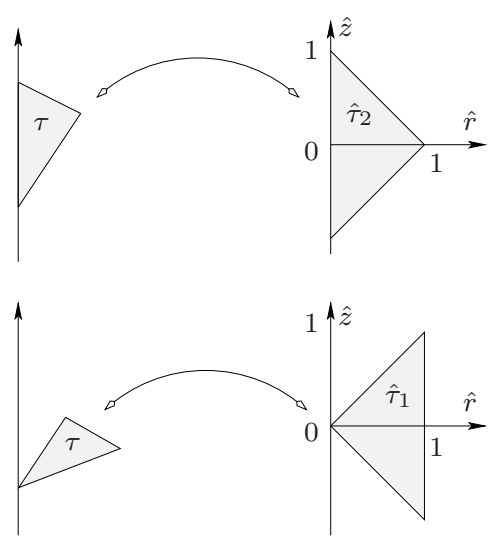

(b)

Figure 1. (a) Illustration of the notations used in some of the proofs in this section. The edges that define degrees of freedom connected to vertices $\mathbf{a}_{i}, \mathbf{a}_{j}$ and $\mathbf{a}_{j+1}$ are marked by thicker lines. (b) The two types of mappings and the two corresponding reference triangles.

though not all edges are degrees of freedom, i.e.,

$$
\int_{e} r \mathbf{v} \cdot \mathbf{t} d s \text { and } \int_{e} r\left(\Pi_{h}^{W} \mathbf{v}\right) \cdot \mathbf{t} d s
$$

are not equal in general.

Additionally, we have the following local norm estimate.

Proposition 5.3. There exists a constant $C$ such that

$$
\left|\Pi_{h}^{W} \mathbf{v}\right|_{H_{1}^{1}(\tau)^{2}} \leq C|\mathbf{v}|_{H_{1}^{1}(\tau)^{2}},
$$

for all mesh triangles $\tau$ in $\mathcal{T}_{h}$ and all $\mathbf{v}$ in $H_{1}^{1}(\tau)^{2}$.

Proof. The result is standard in the case when $\tau$ does not intersect $\Gamma_{0}$. Writing $\left.\Pi_{h}^{W} \mathbf{v}\right|_{\tau}=(a-b z, c+b r)$ on the remaining triangles $\tau$, we have

$$
\begin{aligned}
-2 b \int_{\tau} r d r d z & =\int_{\tau} r \operatorname{curl}_{r z}(a-b z, c+b r) d r d z=\int_{\tau} r \operatorname{curl}_{r z} \Pi_{h}^{W} \mathbf{v} d r d z \\
& =\sigma_{\tau}^{W}\left(\Pi_{h}^{W} \mathbf{v}\right)=\sigma_{\tau}^{W}(\mathbf{v})=\int_{\tau} r \operatorname{curl}_{r z} \mathbf{v} d r d z
\end{aligned}
$$

and hence

$$
\left|\Pi_{h}^{W} \mathbf{v}\right|_{H_{1}^{1}(\tau)^{2}}^{2}=\int_{\tau} r b^{2} d r d z=\frac{\left(\int_{\tau} r \operatorname{curl}_{r z} \mathbf{v} d r d z\right)^{2}}{4 \int_{\tau} r d r d z} \leq C|\mathbf{v}|_{H_{1}^{1}(\tau)^{2}}^{2} .
$$


We also need a projector $\Pi_{h}^{S}$ onto $S_{h}$. Its definition is similar to the rectangular case (4.5); namely, on elements $\tau$ intersecting $\Gamma_{0}$, we define $\left.\left(\Pi_{h}^{S} w\right)\right|_{\tau}$ to be the $L_{1}^{2}(\tau)$-orthogonal projection of $\left.w\right|_{\tau}$ onto the space of constants on $\tau$. On the remaining elements, $\left.\left(\Pi_{h}^{S} w\right)\right|_{\tau}$ is the $L^{2}(\tau)$-orthogonal projection of $w$ onto the space of constants on $\tau$. Then, we have the following analogue of Lemma 4.1 in the rectangular case.

Lemma 5.1. Items (3) and (4) of Lemma 4.1 hold verbatim in the triangular case with the above defined projectors.

Proof. Since the commutative properties are standard for the interpolants based on the standard degrees of freedom, it suffices to verify that they hold on elements $\tau$ with nonempty $\bar{\tau} \cap \bar{\Gamma}_{0}$. On such elements $\tau$, because of the last set of degrees of freedom in (5.2), the identity

$$
\operatorname{curl}_{r z} \Pi_{h}^{W} \mathbf{v}=\Pi_{h}^{S} \operatorname{curl}_{r z} \mathbf{v}
$$

obviously holds. In order to prove the remaining commutative identity, it suffices to show that the degrees of freedom in (5.2) vanish when they are applied to $\left.\left(\Pi_{h}^{W} \operatorname{grad}_{r z} p-\operatorname{grad}_{r z} \Pi_{h}^{V} p\right)\right|_{\tau}$. We need only check $\sigma_{i}^{W}$ and $\sigma_{\tau}^{W}$, as the case of $\sigma_{e}^{W}$ is standard. Now, for any vertex $\mathbf{a}_{i}$ in $\Gamma_{0}$, the definition of $\Pi_{h}^{V}$ gives

$$
\int_{e\left(\mathbf{a}_{i}\right)} r \operatorname{grad}_{r z} \Pi_{h}^{V} p \cdot \mathbf{t} d s=\int_{e\left(\mathbf{a}_{i}\right)} r \operatorname{grad}_{r z} p \cdot \mathbf{t} d s=\int_{e\left(\mathbf{a}_{i}\right)} r \Pi_{h}^{W} \operatorname{grad}_{r z} p \cdot \mathbf{t} d s,
$$

so $\sigma_{i}^{W}\left(\Pi_{h}^{W} \operatorname{grad}_{r z} p-\operatorname{grad}_{r z} \Pi_{h}^{V} p\right)=0$. Finally, if $\tau$ is a triangle in $\mathcal{T}_{h}$ intersecting $\Gamma_{0}$ nontrivially, then

$$
\begin{aligned}
\int_{\tau} r \operatorname{curl}_{r z} \operatorname{grad}_{r z} \Pi_{h}^{V} p d r d z & =0=\int_{\tau} r \operatorname{curl}_{r z} \operatorname{grad}_{r z} p d r d z \\
& =\int_{\tau} r \operatorname{curl}_{r z} \Pi_{h}^{W} \operatorname{grad}_{r z} p d r d z
\end{aligned}
$$

so $\sigma_{\tau}^{W}\left(\Pi_{h}^{W} \operatorname{grad}_{r z} p-\operatorname{grad}_{r z} \Pi_{h}^{V} p\right)=0$. Thus $\Pi_{h}^{W} \operatorname{grad}_{r z} p$ and $\operatorname{grad}_{r z} \Pi_{h}^{V} p$ agree on all the degrees of freedom in $W_{h}$. Hence the commutativity property

$$
\Pi_{h}^{W} \operatorname{grad}_{r z} p=\operatorname{grad}_{r z} \Pi_{h}^{V} p
$$

follows.

Finally, the assertion of the lemma on the boundary conditions is obvious by the construction of the interpolants (where we use the fact that $e\left(\mathbf{a}_{i}\right)$ is contained in $\Gamma_{1}$ if $\mathbf{a}_{i}$ is an endpoint of $\bar{\Gamma}_{0}$ ).

In proving an approximation estimate for the interpolation operator $\Pi_{h}^{W}$, we shall use the next lemma to control the error on edges not having degrees of freedom. In the triangular case, local arguments of the type we used in the rectangular case are not applicable, as the degrees of freedom are defined more globally.

Lemma 5.2. Let $\tau$ be a mesh triangle in $\mathcal{T}_{h}$ having edges $e_{1}, e_{2}, e_{3}$. Set

$$
I_{j}^{r}(\mathbf{u})=\int_{e_{j}} r \mathbf{u} \cdot \mathbf{t} d s \quad \text { and } \quad I_{j}^{1}(\mathbf{u})=\int_{e_{j}} \mathbf{u} \cdot \mathbf{t} d s .
$$

(1) If the edge $e_{3}$ is contained in $\Gamma_{0}$, then

$$
\|\mathbf{u}\|_{L_{1}^{2}(\tau)^{2}}^{2} \leq C\left(h^{2}|\mathbf{u}|_{H_{1}^{1}(\tau)^{2}}^{2}+h^{-1}\left|I_{1}^{r}(\mathbf{u})\right|^{2}+h^{-1}\left|I_{2}^{r}(\mathbf{u})\right|^{2}\right)
$$

for all $\mathbf{u}$ in $H_{1}^{1}(\tau)^{2}$. 
(2) If $e_{3}$ does not intersect $\Gamma_{0}$, but $e_{1}$ and $e_{2}$ have a common endpoint on $\Gamma_{0}$, then

$\|\mathbf{u}\|_{L_{1}^{2}(\tau)^{2}}^{2}+h^{-1}\left|I_{1}^{r}(\mathbf{u})\right|^{2} \leq C\left(h^{2}|\mathbf{u}|_{H_{1}^{1}(\tau)^{2}}^{2}+h^{-1}\left|I_{2}^{r}(\mathbf{u})\right|^{2}+h\left|I_{3}^{1}(\mathbf{u})\right|^{2}\right)$ for all $\mathbf{u}$ in $H_{1}^{1}(\tau)^{2}$.

Proof. The triangle $\tau$ is either a type one or type two triangle (using the terminology in the proof of Proposition [5.2). This proof uses scaling arguments (as in [4]) employing maps from two reference triangles, one for each type of $\tau$. The reference triangles are $\hat{\tau}_{1}$ and $\hat{\tau}_{2}$, defined to be the triangles in the $\hat{r}-\hat{z}$ plane with vertices $\{(0,0),(1,1),(1,-1)\}$ and $\{(0,-1),(1,0),(0,1)\}$, respectively (see Figure 1).

To prove the first estimate, let $\mathbf{F}$ be an affine homeomorphism that maps $\hat{\tau}_{2}$ onto $\tau$ such that $\mathbf{F}$ maps the edge of $\hat{\tau}_{2}$ on the $z$-axis onto $e_{3}$. Map $\mathbf{u}(r, z)$ covariantly to define the function $\hat{\mathbf{u}}(\hat{r}, \hat{z})=J(\mathbf{F})^{t} \mathbf{u}(r, z)$ on $\hat{\tau}_{2}$, where $J(\mathbf{F})$ is the Fréchet derivative of $\mathbf{F}$. If $\lambda_{3}$ is the barycentric coordinate function of $\tau$ that vanishes on $e_{3} \subseteq \Gamma_{0}$, then $r=h_{3} \lambda_{3}$, where $h_{3}$ is the distance from $e_{3}$ to the vertex of $\tau$ opposite of $e_{3}$. Using this, it is easy to show that

$$
\begin{aligned}
\|\mathbf{u}\|_{L_{1}^{2}(\tau)^{2}}^{2} & \leq C h\|\hat{\mathbf{u}}\|_{L_{1}^{2}\left(\hat{\tau}_{2}\right)^{2}}^{2} \\
|\hat{\mathbf{u}}|_{H_{1}^{1}\left(\hat{\tau}_{2}\right)^{2}}^{2} & \leq C h|\mathbf{u}|_{H_{1}^{1}(\tau)^{2}}^{2} \\
\left|\int_{\hat{e}_{i}} \hat{r} \hat{\mathbf{u}} \cdot \hat{\mathbf{t}}_{i} d \hat{s}\right|^{2} & \leq C h^{-2}\left|\int_{e_{i}} r \mathbf{u} \cdot \mathbf{t} d s\right|^{2}, \quad \text { for } i=1 \text { and } 2,
\end{aligned}
$$

where $\hat{e}_{i}=\mathbf{F}^{-1}\left(e_{i}\right)$ and $\hat{\mathbf{t}}_{i}$ denotes the unit tangent on $\hat{e}_{i}$. Therefore, once we show that

$$
C\|\hat{\mathbf{u}}\|_{L_{1}^{2}\left(\hat{\tau}_{2}\right)^{2}}^{2} \leq|\hat{\mathbf{u}}|_{H_{1}^{1}\left(\hat{\tau}_{2}\right)^{2}}^{2}+\left|\hat{I}_{1}^{r}(\hat{\mathbf{u}})\right|^{2}+\left|\hat{I}_{2}^{r}(\hat{\mathbf{u}})\right|^{2}, \quad \text { for all } \hat{\mathbf{u}} \in H_{1}^{1}\left(\hat{\tau}_{2}\right)^{2}
$$

the first inequality of the lemma follows using (5.5)- Here, $\hat{I}_{j}^{r}(\hat{\mathbf{u}})=\int_{\hat{e}_{j}} \hat{r} \hat{\mathbf{u}}$. $\hat{\mathbf{t}} d \hat{s}$.

To establish (5.8), suppose on the contrary that there exists a sequence $\left\{\mathbf{v}_{n}\right\}_{n \in \mathbb{N}}$ in $H_{1}^{1}\left(\hat{\tau}_{2}\right)^{2}$ such that

$$
\frac{1}{n}\left\|\mathbf{v}_{n}\right\|_{L_{1}^{2}\left(\hat{\tau}_{2}\right)^{2}}^{2}>\left|\mathbf{v}_{n}\right|_{H_{1}^{1}\left(\hat{\tau}_{2}\right)^{2}}^{2}+\left|\hat{I}_{1}^{r}\left(\mathbf{v}_{n}\right)\right|^{2}+\left|\hat{I}_{2}^{r}\left(\mathbf{v}_{n}\right)\right|^{2} .
$$

Normalizing, we may assume without loss of generality that $\left\|\mathbf{v}_{n}\right\|_{L_{1}^{2}\left(\hat{\tau}_{2}\right)}=1$ for all $n \in \mathbb{N}$. Thus (5.9) implies that $\lim _{n \rightarrow \infty}\left|\mathbf{v}_{n}\right|_{H_{1}^{1}\left(\hat{\tau}_{2}\right)^{2}}^{2}=0$. In particular, $\left\{\mathbf{v}_{n}\right\}$ is a uniformly bounded sequence in $H_{1}^{1}\left(\hat{\tau}_{2}\right)^{2}$. Consequently, the compact imbedding of $H_{1}^{1}\left(\hat{\tau}_{2}\right)^{2}$ in $L_{1}^{2}\left(\hat{\tau}_{2}\right)^{2}$ [15, Lemma 4.2] yields a subsequence $\left\{\mathbf{v}_{j_{n}}\right\}_{n \in \mathbb{N}}$ converging in $L_{1}^{2}\left(\hat{\tau}_{2}\right)^{2}$. This convergence in $L_{1}^{2}\left(\hat{\tau}_{2}\right)^{2}$ and $\lim _{n \rightarrow \infty}\left|\mathbf{v}_{j_{n}}\right|_{H_{1}^{1}\left(\hat{\tau}_{2}\right)^{2}}=0$ together imply the existence of a $\mathbf{v} \in H_{1}^{1}\left(\hat{\tau}_{2}\right)^{2}$ such that

$$
\lim _{n \rightarrow \infty} \mathbf{v}_{j_{n}}=\mathbf{v} \quad \text { in } H_{1}^{1}\left(\hat{\tau}_{2}\right)^{2} .
$$

Moreover, since $|\mathbf{v}|_{H_{1}^{1}\left(\hat{\tau}_{2}\right)^{2}}=0$, we find that $\mathbf{v}$ is constant. By Lemma A.1. $\hat{I}_{1}^{r}(\cdot)$ and $\hat{I}_{2}^{r}(\cdot)$ are continuous linear functionals on $H_{1}^{1}\left(\hat{\tau}_{2}\right)^{2}$, so we also have that

$$
\hat{I}_{j}^{r}(\mathbf{v})=\left(\mathbf{v} \cdot \hat{\mathbf{t}}_{j}\right) \int_{\hat{e}_{j}} r d \hat{s}=0 \quad \text { for } i=1,2 .
$$

Since the tangent vectors $\hat{\mathbf{t}}_{1}$ and $\hat{\mathbf{t}}_{2}$ are linearly independent, $\mathbf{v} \cdot \hat{\mathbf{t}}_{1}=\mathbf{v} \cdot \hat{\mathbf{t}}_{2}=0$ implies that $\mathbf{v}$ vanishes. But this contradicts $\|\mathbf{v}\|_{L_{1}^{2}\left(\hat{\tau}_{2}\right)^{2}}^{2}=\lim _{n \rightarrow \infty}\left\|\mathbf{v}_{n}\right\|_{L_{1}^{2}\left(\hat{\tau}_{2}\right)^{2}}^{2}=1$, 
so we conclude that (5.8) holds on the reference element $\hat{\tau}_{2}$. Mapping to a target element $\tau$ we complete the proof of the first inequality of the lemma.

The proof of the second inequality of the lemma is similar. For this case, since $\tau$ is a type one triangle, we must use a mapping from the other reference triangle $\hat{\tau}_{1}$. Also, now $r$ is given by a different expression, namely $r=r_{1} \lambda_{1}+r_{2} \lambda_{2}$, where $r_{i}$ denotes the $r$-coordinate of the vertex of $\tau$ opposite of $e_{i}$ and $\lambda_{i}$ is the barycentric coordinate function vanishing on $e_{i}$. But inequalities like (5.5)-(5.7) continue to hold, because $r_{1} / h$ and $r_{2} / h$ are bounded above and below by fixed constants (independent of mesh size, but depending on element angles and on the angle that $\Gamma_{1}$ makes with $\Gamma_{0}$ ). The argument on the reference element $\hat{\tau}_{1}$ by contradiction is analogous to that above.

Lemma 5.3 (Approximation estimates). The projectors defined in the triangular case also satisfy the estimates of Lemma 4.2, i.e., there exists a constant $C$ such that

$$
\begin{aligned}
\left\|\operatorname{grad}_{r z}\left(u-\Pi_{h}^{V} u\right)\right\|_{L_{1}^{2}(D)} & \leq C h|u|_{H_{1}^{2}(D)}, \\
\left\|\mathbf{v}-\Pi_{h}^{W} \mathbf{v}\right\|_{L_{1}^{2}(D)^{2}} & \leq C h|\mathbf{v}|_{H_{1}^{1}(D)^{2}}, \\
\left\|\operatorname{curl}_{r z}\left(\mathbf{v}-\Pi_{h}^{W} \mathbf{v}\right)\right\|_{L_{1}^{2}(D)} & \leq C h\left|\operatorname{curl}_{r z} \mathbf{v}\right|_{H_{1}^{1}(D)}, \\
\left\|w-\Pi_{h}^{S} w\right\|_{L_{1}^{2}(D)} & \leq C h|w|_{H_{1}^{1}(D)},
\end{aligned}
$$

for all $u \in H_{1}^{2}(D), w \in H_{1}^{1}(D)$, and $\mathbf{v} \in H_{1}^{1}(D)^{2}$. For (5.12), we additionally require $\operatorname{curl}_{r z} \mathbf{v} \in H_{1}^{1}(D)$.

Proof. First, (5.13) follows from a result already established in the literature 4, Lemma 5].

As in the proof of Lemma 4.2. (5.12) follows from the commutativity property of Lemma 5.1 and (5.13).

The proof of (5.11) is more involved. Recalling the notations in the proof of Proposition 5.2 (see Figure 1), we first consider a type one triangle $\tau$ with a vertex $\boldsymbol{a}$ on $\Gamma_{0}$. Then there is a sequence of type one triangles $T_{0}, T_{1}, \ldots, T_{n}$, all connected to $\boldsymbol{a}$, with $T_{0}=\tau$, and $T_{n}$ having $e(\boldsymbol{a})$ as an edge. Let $\ell_{j}=\partial T_{j-1} \cap \partial T_{j}$ denote the corresponding sequence of edges connected to $\boldsymbol{a}$. Then, by Lemma 5.2(2) and Proposition 5.3.

$$
\left\|\mathbf{v}-\Pi_{h}^{W} \mathbf{v}\right\|_{L_{1}^{2}(\tau)^{2}}^{2} \leq C\left(h^{2}|\mathbf{v}|_{H_{1}^{1}\left(T_{0}\right)^{2}}^{2}+h^{-1}\left|\int_{\ell_{1}} r\left(\mathbf{v}-\Pi_{h}^{W} \mathbf{v}\right) \cdot \mathbf{t} d s\right|^{2}\right)
$$

where we have used the fact that $\sigma_{e}^{W}$-type degrees of freedom in (5.2) coincide when applied to $\mathbf{v}$ and $\Pi_{h}^{W} \mathbf{v}$. We use Lemma 5.2(2) again, but this time to bound the integral over $\ell_{1}$ appearing above using a seminorm over the next triangle $T_{1}$. Combining with further applications of Lemma 5.2(2) and Proposition 5.3, we have

$$
\begin{aligned}
\left\|\mathbf{v}-\Pi_{h}^{W} \mathbf{v}\right\|_{L_{1}^{2}(\tau)^{2}}^{2} & \leq C\left(h^{2}|\mathbf{v}|_{H_{1}^{1}\left(T_{0} \cup T_{1}\right)^{2}}^{2}+h^{-1}\left|\int_{\ell_{2}} r\left(\mathbf{v}-\Pi_{h}^{W} \mathbf{v}\right) \cdot \mathbf{t} d s\right|^{2}\right) \\
& \leq C\left(h^{2}|\mathbf{v}|_{H_{1}^{1}\left(T_{0} \cup T_{1} \cup \cdots \cup T_{n}\right)^{2}}^{2}+h^{-1}\left|\int_{e(\boldsymbol{a})} r\left(\mathbf{v}-\Pi_{h}^{W} \mathbf{v}\right) \cdot \mathbf{t} d s\right|^{2}\right)
\end{aligned}
$$


The last integral is zero because of the first type of degrees of freedom in (5.2). Thus, if $D_{\boldsymbol{a}}$ denotes the union of all triangles connected to $\boldsymbol{a}$, we have

$$
\left\|\mathbf{v}-\Pi_{h}^{W} \mathbf{v}\right\|_{L_{1}^{2}\left(D_{a}\right)^{2}}^{2} \leq C h^{2}|\mathbf{v}|_{H_{1}^{1}\left(D_{a}\right)^{2}}^{2}
$$

where we have tacitly used the fact that the number $n$ above is bounded by a fixed constant due to the shape regularity of the mesh.

Next consider a type two triangle $\tau$ with edges $e_{1}, e_{2}, e_{3}$ such that $e_{3} \subseteq \Gamma_{0}$. Applying Lemma 5.2(1) and Proposition 5.3.

$$
\left\|\mathbf{v}-\Pi_{h}^{W} \mathbf{v}\right\|_{L_{1}^{2}(\tau)^{2}}^{2} \leq C\left(h^{2}|\mathbf{v}|_{H_{1}^{1}(\tau)^{2}}^{2}+h^{-1}\left|\int_{e_{1} \cup e_{2}} r\left(\mathbf{v}-\Pi_{h}^{W} \mathbf{v}\right) \cdot \mathbf{t} d s\right|^{2}\right) .
$$

The portions of the last integral over $e_{i}$ (for $i=1$ or 2 ) vanish if $e_{i}$ is a degree of freedom. Otherwise, they are bounded as above and we conclude that for any type two triangle $\tau$,

$$
\left\|\mathbf{v}-\Pi_{h}^{W} \mathbf{v}\right\|_{L_{1}^{2}(\tau)^{2}}^{2} \leq C h^{2}|\mathbf{v}|_{H_{1}^{1}\left(D_{\mathbf{x}_{1}} \cup D_{\mathbf{x}_{2}}\right)^{2}}^{2}
$$

where $\mathbf{x}_{i}$ is the vertex of $\tau$ opposite of $e_{i}$.

For triangles $\tau$ not intersecting $\Gamma_{0}$, the estimate

$$
\left\|\mathbf{v}-\Pi_{h}^{W} \mathbf{v}\right\|_{L_{1}^{2}(\tau)^{2}}^{2} \leq C h^{2}\left|\mathbf{v}-\Pi_{h}^{W} \mathbf{v}\right|_{H_{1}^{1}(\tau)^{2}}^{2} \leq C h^{2}|\mathbf{v}|_{H_{1}^{1}(\tau)^{2}}^{2}
$$

follows by standard arguments. Hence by summing over all triangles, and using (5.14), (5.15), or (5.16) as appropriate, we obtain

$$
\left\|\mathbf{v}-\Pi_{h}^{W} \mathbf{v}\right\|_{L_{1}^{2}(D)^{2}} \leq C h|\mathbf{v}|_{H_{1}^{1}(D)^{2}} .
$$

This proves (5.11).

The estimate (5.10) is proved as in the rectangular case using Lemma 5.1 and commutativity. This completes the proof of all the lemmas.

\section{THE DISCRETE MIXED METHOD}

With the tools constructed in the previous sections, we are now able to analyze a discrete mixed method suggested by the variational formulation in Section 3 . The mixed Galerkin method develops approximate solutions in the discrete subspaces considered in the previous sections by requiring them to satisfy the equations of the variational formulation (3.1) in the discrete subspaces. To limit the technicalities in the analysis, we assume throughout this section that the revolution of $D$, namely $\Omega$, is convex.

The mixed Galerkin approximation is the function pair $\left(\mathbf{u}_{h}, p_{h}\right) \in W_{h, \diamond} \times V_{h, \diamond}$ satisfying

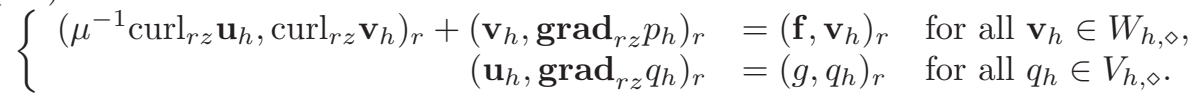

It follows from the next theorem that such a pair is uniquely determined by the above equations. In (6.1), the finite element spaces can be either those defined in Section 4 (for rectangular meshes) or those in Section 5 (for triangular meshes), i.e., $V_{h, \diamond}$ and $W_{h, \diamond}$ are defined by (4.1), where $V_{h}$ and $W_{h}$ can be either as defined in Section 4 or as in Section [5. The analysis of this section treats both cases 
simultaneously, following a standard approach (cf. 16, 6, 7]). Define $W_{h, \diamond}^{0}$ as the subspace of $W_{h, \diamond}$ consisting of discretely divergence-free functions, i.e.,

$$
W_{h, \diamond}^{0}=\left\{\phi_{h} \in W_{h, \diamond}:\left(\phi_{h}, \operatorname{grad}_{r z} p_{h}\right)_{r}=0 \text { for all } p_{h} \in V_{h, \diamond}\right\} .
$$

Then we have the following theorem (cf. Theorem 3.1).

Theorem 6.1. The following holds for the axisymmetric mixed method (6.1):

(1) Discrete inf-sup condition: For all $p_{h}$ in $V_{h, \diamond}$,

$$
C\left\|p_{h}\right\|_{1, r} \leq \sup _{\mathbf{v}_{h} \in W_{h, \diamond}} \frac{\left(\mathbf{v}_{h}, \operatorname{grad}_{r z} p_{h}\right)_{r}}{\left\|\mathbf{v}_{h}\right\|_{r, \text { curl }}} .
$$

(2) Coercivity on the discrete kernel: For all $\mathbf{v}_{h}$ in $W_{h, \diamond}^{0}$,

$$
\left\|\mathbf{v}_{h}\right\|_{r, \text { curl }} \leq C \mu_{1}\left\|\mu^{-1 / 2} \operatorname{curl}_{r z} \mathbf{v}_{h}\right\|_{r} .
$$

(3) Unique solvability: There is a unique $\mathbf{u}_{h}$ in $W_{h, \diamond}$ and a unique $p_{h}$ in $V_{h, \diamond}$ satisfying (6.1).

(4) Optimal error estimates: If $(\mathbf{u}, p)$ in $\mathbf{H}_{r, \diamond}(\operatorname{curl}, D) \times H_{1, \diamond}^{1}(D)$ is the exact solution satisfying (3.1) and $\left(\mathbf{u}_{h}, p_{h}\right)$ in $W_{h, \diamond} \times V_{h, \diamond}$ is the discrete solution satisfying (6.1), then

$$
\left\|\mathbf{u}-\mathbf{u}_{h}\right\|_{\mathbf{H}_{r}(\operatorname{curl}, D)}+\left\|p-p_{h}\right\|_{H_{1}^{1}(D)} \leq C h\left(|\mathbf{u}|_{H_{1}^{1}(D)}+\left|\operatorname{curl}_{r z} \mathbf{u}\right|_{H_{1}^{1}(D)}+|p|_{H_{1}^{2}(D)}\right) .
$$

Here, we have implicitly assumed that $\mathbf{u}$ and $p$ are smooth enough so that the above norms make sense.

Proof. (1) The proof of the inf-sup condition is similar to the proof of the exact infsup condition in Theorem 3.1(10. The only change is that we use the discrete version of $\operatorname{grad}_{r z} H_{1, \diamond}^{1}(D) \subseteq \mathbf{H}_{r, \diamond}(\operatorname{curl}, D)$, namely that for all $p_{h}$ in $V_{h, \diamond}$, the gradient $\operatorname{grad}_{r z} p_{h}$ is in $W_{h, \diamond}$.

(2) The proof of this inequality is an adaptation of the proof of Proposition 5.1(III) of [10]. Let $p \in H_{1, \diamond}^{1}(D)$ be the unique solution (cf. [11]) to

$$
\left(\operatorname{grad}_{r z} p, \operatorname{grad}_{r z} q\right)_{r}=\left(\mathbf{v}_{h}, \operatorname{grad}_{r z} q\right)_{r} \quad \text { for all } q \in H_{1, \diamond}^{1}(D),
$$

and put

$$
\mathbf{w}:=\mathbf{v}_{h}-\operatorname{grad}_{r z} p .
$$

Define the revolved vector field $\breve{\mathbf{w}}=w_{r} \mathbf{e}_{r}+w_{z} \mathbf{e}_{z}$ on $\Omega$. By the same argument given in the proof of Theorem 3.1(2), $\breve{\mathbf{w}}$ is in $H_{0}(\operatorname{curl}, \Omega)$ and satisfies

$$
(\breve{\mathbf{w}}, \operatorname{grad} q)_{L^{2}(\Omega)}=0 \quad \text { for all } q \in H_{0}^{1}(\Omega),
$$

i.e., $\breve{\mathbf{w}}$ is divergence free. Now, a well known imbedding of $H_{0}(\mathbf{c u r l}, \Omega) \cap H(\operatorname{div}, \Omega)$ into $H^{1}(\Omega)^{3}$ for convex $\Omega$ [10, Proposition I.3.1] yields

$$
\|\breve{\mathbf{w}}\|_{H^{1}(\Omega)^{3}} \leq C\|\operatorname{curl} \breve{\mathbf{w}}\|_{L^{2}(\Omega)^{3}}=C\left\|\operatorname{curl} \breve{\mathbf{v}}_{h}\right\|_{L^{2}(\Omega)^{3}},
$$

where, in the last step, we used curl $\breve{\mathbf{w}}=\operatorname{curl} \breve{\mathbf{v}}_{h}$. By [3, Proposition 3.17], w $=$ $\left(w_{r}, w_{z}\right)$ is in $H_{-}^{1}(D) \times H_{1}^{1}(D)$ (and is bounded by an accompanying norm estimate). Hence,

$$
\|\mathbf{w}\|_{H_{1}^{1}(D)^{2}} \leq\|\mathbf{w}\|_{H_{-}^{1}(D) \times H_{1}^{1}(D)} \leq C\|\breve{\mathbf{w}}\|_{H^{1}(\Omega)^{3}} \leq C\left\|\operatorname{curl} \breve{\mathbf{v}}_{h}\right\|_{L^{2}(\Omega)^{3}} .
$$

To prove the required inequality for $\mathbf{v}_{h}$, we use the above estimates for $\mathbf{w}$. Since w is in $H_{1}^{1}(D)^{2}$, the projection $\Pi_{h}^{W} \mathbf{w}$ is well defined by Lemma 4.1)(2) (rectangular case) or Proposition 5.2 (triangular case). Obviously, $\operatorname{grad}_{r z} p=\mathbf{v}_{h}-\mathbf{w}$ implies 
that on each element $\tau,\left.\left(\operatorname{grad}_{r z} p\right)\right|_{\tau}$ is in $H_{1}^{1}(\tau)^{2}$, so it possesses enough regularity for the application of $\Pi_{h}^{W}$. Furthermore, since $\Pi_{h}^{W} \mathbf{v}_{h}=\mathbf{v}_{h}$,

$$
\begin{aligned}
\mathbf{v}_{h} & =\Pi_{h}^{W} \mathbf{w}+\Pi_{h}^{W} \operatorname{grad}_{r z} p \\
& =\Pi_{h}^{W} \mathbf{w}+\operatorname{grad}_{r z} \Pi_{h}^{V} p,
\end{aligned}
$$

using the commutativity properties of Lemma 4.1 (rectangular case) or Lemma 5.1 (triangular case). Now since $\mathbf{v}_{h}$ is $L_{1}^{2}(D)$-orthogonal to $\operatorname{grad}_{r z} V_{h, \diamond}$, the above equation implies

$$
\left(\mathbf{v}_{h}, \mathbf{v}_{h}\right)_{r}=\left(\Pi_{h}^{W} \mathbf{w}, \mathbf{v}_{h}\right)_{r}
$$

which, by the Cauchy-Schwarz inequality, gives

$$
\left\|\mathbf{v}_{h}\right\|_{L_{1}^{2}(D)^{2}} \leq\left\|\Pi_{h}^{W} \mathbf{w}\right\|_{L_{1}^{2}(D)^{2}} .
$$

Combining this with the approximation properties of Lemma 4.2 or Lemma 5.3

$$
\begin{aligned}
\left\|\mathbf{v}_{h}\right\|_{L_{1}^{2}(D)^{2}} \leq\left\|\Pi_{h}^{W} \mathbf{w}\right\|_{L_{1}^{2}(D)^{2}} & \leq\left\|\Pi_{h}^{W} \mathbf{w}-\mathbf{w}\right\|_{L_{1}^{2}(D)^{2}}+\|\mathbf{w}\|_{L_{1}^{2}(D)^{2}} \\
& \leq C h|\mathbf{w}|_{H_{1}^{1}(D)}+\|\mathbf{w}\|_{L_{1}^{2}(D)^{2}} \\
& \leq C\left\|\operatorname{curl}_{r z} \mathbf{v}_{h}\right\|_{L_{1}^{2}(D)},
\end{aligned}
$$

where in the last step we have used (6.3). This proves the coercivity on the discrete kernel.

(3) The unique solvability is a well known consequence of (1) and (2) (cf. 7, 10]).

(4) The error estimate now follows from the Babuška-Brezzi theory [7, 10]. Indeed, (1) and (2) imply quasioptimality, i.e.,

$$
\begin{aligned}
\| \mathbf{u}- & \mathbf{u}_{h}\left\|_{\mathbf{H}_{r}(\operatorname{curl}, D)}+\right\| p-p_{h} \|_{H_{1}^{1}(D)} \\
& \leq C\left(\inf _{\mathbf{v}_{h} \in W_{h, \diamond}}\left\|\mathbf{u}-\mathbf{v}_{h}\right\|_{\mathbf{H}_{r}(\operatorname{curl}, D)}+\inf _{q_{h} \in V_{h, \diamond}}\left\|p-q_{h}\right\|_{H_{1}^{1}(D)}\right) \\
& \leq C\left(\left\|\mathbf{u}-\Pi_{h}^{W} \mathbf{u}\right\|_{\mathbf{H}_{r}(\operatorname{curl}, D)}+\left\|p-\Pi_{h}^{V} p\right\|_{H_{1}^{1}(D)}\right) .
\end{aligned}
$$

Here we used the previously noted fact that the projectors $\Pi_{h}^{W}$ and $\Pi_{h}^{V}$ preserve zero boundary conditions (see Lemma 4.14(4) or Lemma 5.1), so that $\Pi_{h}^{W} \mathbf{u}$ and $\Pi_{h}^{V} p$ are indeed in $W_{h, \diamond}$ and $V_{h, \diamond}$, respectively. Estimating the right hand side above by Lemma 4.2 (rectangular case) or Lemma 5.3 (triangular case) and applying a Poincaré inequality completes the proof of the theorem.

\section{Numerical Results}

In this section, we verify that the finite element convergence rates predicted by our analysis are achieved in a numerical example. We also make suggestions on how to iteratively solve the discrete matrix system. As in the previous section, the finite element spaces $V_{h, \diamond}$ and $W_{h, \diamond}$ can be defined on rectangular or triangular meshes. Define the operators $A_{h}: W_{h, \diamond} \rightarrow\left(W_{h, \diamond}\right)^{\prime}$ and $B_{h}: W_{h, \diamond} \rightarrow\left(V_{h, \diamond}\right)^{\prime}$ by

$$
\begin{array}{ll}
A_{h} \mathbf{v}_{h}\left(\mathbf{w}_{h}\right)=\left(\mu^{-1} \operatorname{curl}_{r z} \mathbf{v}_{h}, \operatorname{curl}_{r z} \mathbf{w}_{h}\right)_{r} & \text { for all } \mathbf{v}_{h}, \mathbf{w}_{h} \in W_{h, \diamond}, \\
B_{h} \mathbf{v}_{h}\left(q_{h}\right)=\left(\mathbf{v}_{h}, \operatorname{grad}_{r z} q_{h}\right)_{r} & \text { for all } \mathbf{v}_{h} \in W_{h, \diamond}, q_{h} \in V_{h, \diamond} .
\end{array}
$$

Let $\mathrm{A}_{h}$ and $\mathrm{B}_{h}$ denote the matrix representations of the operators $A_{h}$ and $B_{h}$, respectively, in the standard bases for $V_{h, \diamond}$ and $W_{h, \diamond}$. Then (6.1) is rewritten as the linear system

$$
\mathrm{C}_{h} \mathrm{x}_{h}=\mathrm{b}_{h}
$$


TABLE 7.1. Constant coefficient $(\mu \equiv 1)$ on the unit square $D$.

\begin{tabular}{|c|c|c|c|c|c|c|}
\hline level & $L_{1}^{2}$ error of $\left(A_{r}, A_{z}\right)$ & Order & PCG & Points & Elements & Unknowns \\
\hline \hline 1 & 0.0851831 & & 8 & 49 & 72 & 240 \\
\hline 2 & 0.0515349 & 0.725 & 8 & 169 & 288 & 1032 \\
\hline 3 & 0.0255215 & 1.014 & 8 & 625 & 1152 & 4128 \\
\hline 4 & 0.0126821 & 1.009 & 8 & 2401 & 4608 & 16368 \\
\hline 5 & 0.0063197 & 1.004 & 8 & 9409 & 18432 & 65040 \\
\hline 6 & 0.0031543 & 1.003 & 8 & 37249 & 73728 & 259152 \\
\hline 7 & 0.0015757 & 1.001 & 8 & 148225 & 294912 & 1034448 \\
\hline
\end{tabular}

where

$$
\mathrm{C}_{h}=\left[\begin{array}{cc}
\mathrm{A}_{h} & \mathrm{~B}_{h}^{t} \\
\mathrm{~B}_{h} & 0
\end{array}\right], \quad \mathrm{x}_{h}=\left[\begin{array}{l}
\mathrm{u}_{h} \\
\mathrm{p}_{h}
\end{array}\right], \quad \text { and } \mathrm{b}_{h}=\left[\begin{array}{c}
\mathrm{f}_{h} \\
\mathrm{~g}_{h}
\end{array}\right] .
$$

Here, $\mathbf{u}_{h}$ and $\mathbf{p}_{h}$ denote the vectors of coefficients in the expansions of $\mathbf{u}_{h}$ and $p_{h}$ in the standard bases, respectively, and the right hand side vector $b_{h}$ is computed from the right hand side of (6.1) as usual. Note that the matrix $C_{h}$ is symmetric but indefinite.

For practical problems with a large number of degrees of freedom, since the matrix system (7.1) is sparse, we solve (7.1) using a preconditioned iterative method. To precondition the linear system (7.1), we employ multigrid methods on the spaces $V_{h, \diamond}$ and $W_{h, \diamond}$ and forms coming from the $\mathbf{H}_{r}(\operatorname{curl}, D)$ and $H_{1}^{1}(D)$ inner-products, respectively. The uniform convergence of a $\mathrm{V}$-cycle multigrid with standard GaussSeidel smoothing was proved in [11. For the Nédélec subspace $W_{h, \diamond}$ of $\mathbf{H}_{r}(\operatorname{curl}, D)$, we use the V-cycle multigrid with Arnold-Falk-Winther smoothing [2]. Let $\mathrm{M}_{V}$ and $\mathrm{M}_{W}$ denote the matrices representing the multigrid V-cycles acting on the matrices with entries given by the inner products of basis functions in $V_{h, \diamond}$ and $W_{h, \diamond}$, respectively. Instead of the indefinite system (7.1), we solve the symmetric positive definite system

$$
\left(\mathrm{A}_{h}+\mathrm{B}_{h}^{t} \mathrm{M}_{V} \mathrm{~B}_{h}\right) \mathrm{u}_{h}=\mathrm{f}_{h}+\mathrm{B}_{h}^{t} \mathrm{M}_{V} \mathrm{~g}_{h}
$$

by the preconditioned conjugate gradient method (PCG), with $\mathrm{M}_{W}$ as a preconditioner. The system (7.2) is obtained from (7.1) by replacing the zero block with $-\mathrm{M}_{V}^{-1}$, since $\mathrm{p}_{h}=0$. In all the numerical experiments reported below, the termination condition for the PCG iterations is the reduction of the energy norm of the residual by a factor of $10^{-12}$.

In Table 7.1, we list the $L_{1}^{2}(D)^{2}$-norm of the error in approximating $\left(A_{r}, A_{z}\right)$ on a sequence of uniformly refined, unstructured meshes of the unit square. These results are for the constant coefficient $\mu \equiv 1$ and data

$$
\begin{aligned}
& \mathbf{f}=\left(\pi^{2} \sin \pi z, \frac{\pi}{r}(\cos \pi z-\cos \pi r)+\pi^{2} \sin \pi r\right), \\
& g=\frac{\sin \pi z}{r} .
\end{aligned}
$$

In this case, the exact solution is the bounded and smooth vector field $\left(A_{r}, A_{z}\right)=$ $(\sin \pi z, \sin \pi r)$. The coarsest mesh (level 1 ) has a mesh size of approximately $1 / 6$. Each refinement of a mesh is performed by connecting the midpoints of edges of each mesh triangle, so the finest mesh (level 7 ) is roughly of size $1 / 384$. The third 
TABLE 7.2. PCG iteration counts for discontinuous coefficients (7.3) and (7.4) on the unit square $D$.

\begin{tabular}{|c|c|c|c|c|c|}
\hline level & PCG for (7.3) & PCG for (7.4) & Points & Elements & Unknowns \\
\hline \hline 1 & 15 & 23 & 45 & 64 & 246 \\
\hline 2 & 16 & 23 & 153 & 256 & 954 \\
\hline 3 & 17 & 23 & 561 & 1024 & 3714 \\
\hline 4 & 17 & 23 & 2145 & 4096 & 14610 \\
\hline 5 & 17 & 23 & 8385 & 16384 & 57906 \\
\hline 6 & 17 & 23 & 33153 & 65536 & 230514 \\
\hline 7 & 17 & 26 & 131841 & 262144 & 919794 \\
\hline
\end{tabular}

column lists the observed order of convergence (the entry in the $j^{\text {th }}$ row of this column is equal to $\log _{2}\left(\varepsilon_{j-1} / \varepsilon_{j}\right)$, where $\varepsilon_{j}$ is the $L_{1}^{2}(D)^{2}$-norm of the error at level $j$ ). We observe the theoretically predicted first-order convergence rate.

The effectiveness of the iterative solution technique by multigrid preconditioning is also evident from Table 7.1 - the fourth column of the table lists the number of PCG iterations taken to satisfy the termination condition. Observe that only 8 iterations are needed in all cases. Table 7.2 lists the PCG iteration counts for the following two discontinuous coefficients:

$$
\begin{gathered}
\mu= \begin{cases}(1+\sin r) / 2, & \text { if } z>1 / 2, \\
1, & \text { otherwise, }\end{cases} \\
\mu= \begin{cases}10^{4}, & \text { if } z>1 / 2, \\
1, & \text { otherwise. }\end{cases}
\end{gathered}
$$

The finest mesh size (level 7) is $1 / 256$. For both of these discontinuous coefficients, we observe that the preconditioned iterative solution technique works well.

\section{Appendix A. A trace Result}

In this appendix we show a trace result for $H_{1}^{1}(\tau)$ for a triangle $\tau$ connected to $\Gamma_{0}$. As seen from Figure 1, $\tau$ can be either of type one or type two. If $h_{\tau}$ and $\rho_{\tau}$ are the diameters of $\tau$ and the largest circle inscribed in $\tau$, respectively, then the ratio $\rho_{\tau} / h_{\tau}$ measures the shape regularity of the triangle. We expect the trace inequality to have a constant $C_{\text {trc }}$ dependent on $\rho_{\tau} / h_{\tau}$. This dependence can be translated into a dependence on the interior angles of $\tau$. Our purpose here is to show that the trace constant also depends on the angle $\tau$ makes with the $z$-axis and that the weights in the trace inequality are different for type one and type two triangles. Of the two nonzero angles that the edges of $\tau$ make with the $z$-axis, let $\theta$ be the one with the smaller sine.

Lemma A.1. Suppose e is an edge of $\tau$ that intersects $\bar{\Gamma}_{0}$ at one and only one point. Then there is a constant $C_{\operatorname{trc}}$ depending on the shape regularity constant $\rho_{\tau} / h_{\tau}$, but otherwise independent of $\tau$, such that for all $v$ in $H_{1}^{1}(\tau)$, we have the following:

(1) If $\tau$ is a type one triangle, then

$$
\frac{1}{|e|}\|v\|_{L_{2}^{2}(e)}^{2} \leq C_{\operatorname{trc}} \frac{h_{\tau}}{\sin \theta}\left(\frac{1}{h_{\tau}^{2}}\|v\|_{L_{1}^{2}(\tau)}^{2}+|v|_{H_{1}^{1}(\tau)}^{2}\right) .
$$


(2) If $\tau$ is a type two triangle, then

$$
\frac{1}{|e|}\|v\|_{L_{1}^{2}(e)}^{2} \leq C_{\operatorname{trc}} \frac{1}{\sin \theta}\left(\frac{1}{h_{\tau}^{2}}\|v\|_{L_{1}^{2}(\tau)}^{2}+|v|_{H_{1}^{1}(\tau)}^{2}\right) .
$$

Proof. It suffices to prove the stated inequalities for $v$ in $\mathcal{D}(\bar{\tau})$ because of the density of $\mathcal{D}(\bar{\tau})$ in $H_{1}^{1}(\tau)$ [13]. First, consider the type one case. Let the type one reference triangle $\hat{\tau}_{1}$ (see Figure 1) be mapped onto $\tau$ by an affine map $\mathbf{F}$. Without loss of generality, assume that $e=\mathbf{F}(\hat{e})$, where $\hat{e}$ is the edge of $\hat{\tau}_{1}$ in the first quadrant of the $\hat{r}-\hat{z}$ plane. If $\hat{v}=v \circ \mathbf{F}$, then the following estimates are easy to verify:

$$
\begin{aligned}
\frac{1}{|e|}\|v\|_{L_{2}^{2}(e)}^{2} & \leq \hat{c} \bar{r}_{\tau}^{2}\|\hat{v}\|_{L_{2}^{2}(\hat{e})}^{2}, \\
\|\hat{v}\|_{L_{1}^{2}\left(\hat{\tau}_{1}\right)}^{2} & \leq \frac{\hat{c}}{h_{\tau}^{2} \underline{r}_{\tau}}\|v\|_{L_{1}^{2}(\tau)}^{2}, \\
|\hat{v}|_{H_{1}^{1}\left(\hat{\tau}_{1}\right)}^{2} & \leq \frac{\hat{c}}{\underline{r}_{\tau}}|v|_{H_{1}^{1}(\tau)}^{2},
\end{aligned}
$$

where $\bar{r}_{\tau}$ and $\underline{r}_{\tau}$ denote the maximum and minimum of the $r$-coordinate values of the two vertices of $\tau$ not on $\bar{\Gamma}_{0}$, respectively, and $\hat{c}$ depends only on the shape regularity constant. Note that $\bar{r}_{\tau} / \underline{r}_{\tau} \leq C_{\mathrm{trc}} / \sin \theta$. We will now show that

$$
\frac{1}{\sqrt{2}}\|\hat{v}\|_{L_{2}^{2}(\hat{e})}^{2} \leq\|\hat{v}\|_{L_{1}^{2}\left(\hat{\tau}_{1}\right)}^{2}+2\left\|\partial_{z} \hat{v}\right\|_{L_{3}^{2}\left(\hat{\tau}_{1}\right)}^{2} .
$$

Once this is established, the required inequality follows from A.1 A.3.

To prove (A.4), we consider a smooth function $v$ on $\hat{\tau}_{1}$ (dropping the accent from $\hat{r}, \hat{z}$, and $\hat{v}$ for convenience) and use the fundamental theorem of calculus to write

$$
v(r, r)-v(r, z)=\int_{z}^{r} \partial_{z} v(r, \zeta) d \zeta
$$

so that

$$
|v(r, r)|^{2} \leq 2|v(r, z)|^{2}+2\left|\int_{z}^{r} \partial_{z} v(r, \zeta) d \zeta\right|^{2}
$$

Integrating over $z$ from $-r$ to $r$,

$$
\begin{aligned}
r|v(r, r)|^{2} & \leq \int_{-r}^{r}|v(r, z)|^{2} d z+\int_{-r}^{r}\left|\int_{z}^{r} \partial_{z} v(r, \zeta) d \zeta\right|^{2} d z \\
& \leq \int_{-r}^{r}|v(r, z)|^{2} d z+2 r^{2} \int_{-r}^{r}\left|\partial_{z} v(r, \zeta)\right|^{2} d \zeta .
\end{aligned}
$$

Multiplying by $r$ and integrating over $r$ from 0 to 1 yields (A.4). This completes the proof for the type one case.

The proof of the type two case proceeds similarly, but using the reference triangle $\hat{\tau}_{2}$ instead (see Figure 11). Again, we denote by $\hat{e}$ the edge of $\hat{\tau}_{2}$ in the first quadrant of the $\hat{r}-\hat{z}$ plane. As in the previous case,

$$
v(r, 1-r)-v(r, z)=\int_{z}^{1-r} \partial_{z} v(r, \zeta) d \zeta
$$

implies

$$
|v(r, 1-r)|^{2} \leq 2|v(r, z)|^{2}+4 \int_{z}^{1-r}\left|\partial_{z} v(r, \zeta)\right|^{2} d \zeta
$$


Integrating over $-1+r<z<1-r$, we have

$$
(1-r)|v(r, 1-r)|^{2} \leq \int_{-1+r}^{1-r}|v(r, z)|^{2} d z+4 \int_{-1+r}^{1-r}\left|\partial_{z} v(r, \zeta)\right|^{2} d \zeta .
$$

Unlike the previous case, we now observe the presence of the factor $(1-r)$ above. Upon further integration over $0<r<1$, this will result in an unwanted degenerate weight. Therefore, we integrate only over $0<r<1 / 2$, obtaining

$$
\begin{aligned}
\frac{1}{2} \int_{0}^{1 / 2} r|v(r, 1-r)|^{2} d r & \leq \int_{0}^{1 / 2} r(1-r)|v(r, 1-r)|^{2} d r \\
& \leq\|v\|_{L_{1}^{2}\left(\hat{\tau}_{2}\right)}^{2}+4\left\|\partial_{z} v\right\|_{L_{1}^{2}\left(\hat{\tau}_{2}\right)}^{2} .
\end{aligned}
$$

For the remaining piece of $\hat{e}$ where $1 / 2<r<1$, we integrate along the line segment $\{(s, s-2 r+1): r-1 / 2<s<r\}$ of length $\sqrt{2} / 2$, perpendicular to $\hat{e}$ at $(r, 1-r)$. This gives

$$
|v(r, 1-r)|^{2} \leq 2|v(s, s-2 r+1)|^{2}+\left|\int_{s}^{r}(1,1) \cdot \operatorname{grad}_{r z} v(\zeta, \zeta-2 r+1) d \zeta\right|^{2}
$$

Integrating over $r-1 / 2<s<r$ and then over $1 / 2<r<1$, we have

$$
\frac{1}{2} \int_{1 / 2}^{1} r|v(r, 1-r)|^{2} d r \leq 2\|v\|_{L_{1}^{2}\left(\hat{\tau}_{2}\right)}^{2}+\frac{1}{2}|v|_{H_{1}^{1}\left(\hat{\tau}_{2}\right)}^{2} .
$$

Combining (A.5) and A.6 yields

$$
\frac{1}{\sqrt{2}}\|v\|_{L_{1}^{2}(\hat{e})}^{2} \leq 6\|v\|_{L_{1}^{2}\left(\hat{\tau}_{2}\right)}^{2}+9|v|_{H_{1}^{1}\left(\hat{\tau}_{2}\right)}^{2} .
$$

The scaling argument to complete the proof is similar to the type one case. The only difference is that the norm on $L_{1}^{2}(e)$ gives one less factor of $\bar{r}_{\tau}$ than the norm on $L_{2}^{2}(e)$, resulting in one less factor of $h_{\tau}$.

\section{REFERENCES}

1. C. Amrouche, C. Bernardi, M. Dauge, and V. Girault, Vector potentials in threedimensional non-smooth domains, Math. Methods Appl. Sci., 21 (1998), pp. 823-864. MR $1626990(99 \mathrm{e}: 35037)$

2. D. N. Arnold, R. S. Falk, And R. Winther, Multigrid in $H$ (div) and $H$ (curl), Numer. Math., 85 (2000), pp. 197-217. MR1754719 (2001d:65161)

3. F. Assous, P. Ciarlet, JR., AND S. LABRUnie, Theoretical tools to solve the axisymmetric Maxwell equations, Math. Methods Appl. Sci., 25 (2002), pp. 49-78. MR1874449 (2002j:78008)

4. Z. Belhachmi, C. Bernardi, and S. Deparis, Weighted Clement operator and application to the finite element discretization of the axisymmetric Stokes problem, Numer. Math., 105 (2006), pp. 217-247. MR2262757

5. C. Bernard, M. Dauge, And Y. Maday, Spectral methods for axisymmetric domains, vol. 3 of Series in Applied Mathematics (Paris), Gauthier-Villars, Éditions Scientifiques et Médicales Elsevier, Paris, 1999. MR1693480(2000h:65002)

6. D. BofFI, Fortin operator and discrete compactness for edge elements, Numer. Math., 87 (2000), pp. 229-246. MR1804657 (2001k:65168)

7. F. Brezzi And M. Fortin, Mixed and hybrid finite element methods, vol. 15 of Springer Series in Computational Mathematics, Springer-Verlag, New York, 1991. MR.1115205 (92d:65187)

8. O. Chinellato, The Complex-Symmetric Jacobi-Davidson Algorithm and its Application to the Computation of some Resonance Frequencies of Anisotropic Lossy Axisymmetric Cavities, Dissertation ETH No. 16243, Swiss Federal Institute of Technology, Zürich, 2005.

9. D. M. Copeland And J. E. Pasciak, A least-squares method for axisymmetric div-curl systems, Numer. Linear Algebra Appl., 13 (2006), pp. 733-752. MR2266104 
10. V. Girault And P.-A. Raviart, Finite element approximation of the Navier-Stokes equations, vol. 749 of Lecture Notes in Mathematics, Springer-Verlag, Berlin, 1979. MR548867 (83b:65122)

11. J. GOPALAKRISHNAN AND J. E. PASciak, The convergence of V-cycle multigrid algorithms for axisymmetric Laplace and Maxwell equations, Math. Comp., 75 (2006), pp. 1697-1719. MR 2240631 (2007g:65116)

12. F. KIKUCHI, Numerical analysis of electrostatic and magnetostatic problems, Sugaku Expositions, 6 (1993), pp. 33-51. MR 1222042

13. A. Kufner, Weighted Sobolev spaces, A Wiley-Interscience Publication, John Wiley \& Sons Inc., New York, 1985. Translated from the Czech. MR802206 (86m:46033)

14. P. Lacoste And J. Gay, A new family of finite elements for Maxwell-Fourier's equations, in Mathematical and numerical aspects of wave propagation phenomena (Strasbourg, 1991), SIAM, Philadelphia, PA, 1991, pp. 746-749. MR.1106041 (92c:65135)

15. B. Mercier And G. Raugel, Résolution d'un problème aux limites dans un ouvert axisymétrique par éléments finis en $r$, $z$ et séries de Fourier en $\theta$, RAIRO Anal. Numér., 16 (1982), pp. 405-461. In French. MR684832 (84g:65154)

16. P. Monk, Finite element methods for Maxwell's equations, Numerical Mathematics and Scientific Computation, Oxford University Press, New York, 2003. MR2059447 (2005d:65003)

17. J.-C. NÉdÉLEC, Mixed Finite Elements in $\mathbb{R}^{3}$, Numer. Math., 35 (1980), pp. 315-341. MR $592160(81 \mathrm{k}: 65125)$

18. C. Weber, A local compactness theorem for Maxwell's equations, Math. Methods Appl. Sci., 2 (1980), pp. 12-25. MR561375 (81f:78005)

Johann Radon Institute for Computational and Applied Mathematics, Austrian Academy of Sciences, Altenbergerstrasse 69, A-4040 Linz, Austria

E-mail address: dylan.copeland@ricam.oeaw.ac.at

Department of Mathematics, University of Florida, Gainesville, Florida 32611-8105

E-mail address: jayg@math.ufl.edu

Department of Mathematics, Texas A\&M University, College Station, Texas 77843

E-mail address: pasciak@math.tamu.edu 